Educación

\title{
FORMACIÓN DE ANESTESISTAS EN CHILE CÓMO HA EVOLUCIONADO LA FORMACIÓN EN NUESTRA ESPECIALIDAD EN LOS ÚLTIMOS 40 AÑOS
}

\author{
Renato Chacón A. ${ }^{1}$
}

Key words: Education.

"En estos momentos hay 249 anestesiólogos en formación en el país: 91 en primer año, 79 en segundo año y 78 en tercer año. Ellos se distribuyen en 13 programas de post título universitarios y un programa no universitario".

Al comenzar a redactar este artículo escribí este primer párrafo que de acuerdo a la información con que contaba, resumía la realidad gruesa de la formación de anestesistas en Chile y me permitía desarrollar el tema a partir de cifras que probablemente muy pocos conocen.

Sin embargo, mi información era incompleta, el aumento explosivo de programas de post título en anestesiología incluía dos más que yo no tenía considerados. Por tanto, dicho párrafo ahora debe señalar:

"En estos momentos hay 256 anestesiólogos en formación en el país: 98 en primer año, 80 en segundo año y 78 en tercer año. Ellos se distribuyen en 15 programas de post título universitario y un programa no universitario".

Al darme cuenta de este inesperado aumento de becados y de programas, no pude evitar recordar la película "Los Gremlins", en que unas criaturas se reproducen sin control al ponerse en contacto con el agua. Más aún, fui a Wikipedia y en relación a esta película encontré la siguiente frase: "La historia es narrada, en forma de cuento con moraleja, por el padre de un adolescente al que regalan por navidad una extraña mascota llamada Gizmo. La criatura pertenece a una especie animal que bajo determinadas circunstancias se transforma en un pequeño monstruo muy destructivo. Por lo tanto, la mascota exige de su cuidador una gran responsabilidad, moraleja final de la historia".

Como la alusión a esta película pudiera parecer algo peyorativa, me preocupa dejar en claro que yo no tengo una posición contraria a la creación de nuevos programas de formación de anestesiólogos. Creo que son necesarios, así como es necesario que los "antiguos" programas potencien su capacidad formativa, a la luz del agudo déficit de especialistas que aflige a prácticamente todos los hospitales públicos y a muchas clínicas privadas del país.

Sin embargo, eso no obsta el que la creación de estos nuevos programas debe ser programada con una importante cuota de responsabilidad.

En tal sentido, me parece que intentar mantener el statu quo desconociendo la necesidad de formar más anestesistas, es tan peligroso para la especialidad como iniciar una formación indiscriminada y de dudosa calidad.

Por lo mismo, me parece que no existe posibilidad de proyectar responsablemente la formación de anestesistas sin conocer las cifras reales que mueven el desafío. Dentro de ellas, existe una excesivamente manoseada: el déficit real de anestesistas en el país.

En los últimos años hemos escuchado al menos 5 cifras distintas de déficit de anestesistas, planteadas por diferentes instituciones, cosa que no hace

Anestesiólogo Hospital de Urgencia Asistencia Pública. Anestesiólogo Clínica Dávila.

Profesor Adjunto II Universidad de Santiago.

Profesor Asociado Universidad de los Andes. 
más que refrendar que todas ellas son erróneas. En consecuencia, creo imprescindible que la Sociedad de Anestesiología de Chile se involucre seriamente en un estudio que determine dicha cifra en forma real.

En este artículo se analiza la otra variable de la ecuación: la formación de especialistas y su evolución durante los últimos 40 años. En él ustedes conocerán las cifras de la formación actual de anestesistas y algunas características singulares de cada programa.

Sin embargo, aspiro a que el artículo también les permita encontrarse con la historia. Muchos de ustedes se identificarán en alguna de las nóminas o podrán reconocer a aquellos que posteriormente fueron sus docentes. En dichas nóminas aparecen como becados aquellos que posteriormente se convirtieron en los líderes de sus Servicios de Anestesiología y también aquellos que fueron reconocidos posteriormente como Maestros de la Anestesiología Chilena.

Es muy probable que los listados estén incompletos, por lo que si cometí omisiones, les pido las disculpas del caso. En mi favor solo el argumento de que construí estos archivos en base a lo que los propios programas me pudieron entregar como información. Al fin y al cabo este documento reúne 919 nombres de anestesiólogos formados en programas oficiales a partir de la década del 60 .

\section{CIFRAS BÁSICAS}

Parto en los mismos números: en estos momentos hay 256 anestesiólogos en formación en el país; 98 en primer año, 80 en segundo año y 78 en tercer año de beca.

Ellos se distribuyen en 15 programas de post título universitarios y un plan de formación no universitario radicado en el Servicio de Salud Metropolitano Sur-Oriente.

Cinco de estos programas iniciaron su funcionamiento hace más de 15 años, uno lo hizo el año 2005, dos el año 2009, dos el año 2011, tres el año 2013 y tres el año 2014, lo cual da cuenta del explosivo aumento de estos durante los últimos años.

Siete de ellos $(43,75 \%)$ se encuentran acreditados por diferentes períodos, de acuerdo al sistema vigente, a través de la Agencia de Acreditación de Programas Universitarios de Especialidades Médicas - APICE.

Ocho programas tienen su asiento en Santiago y los restantes ocho en provincia, todos en ciudades del sur del país.

La Tabla 1 resume estos datos.

El desarrollo de estos programas a través del tiempo no solo constituye un encuentro con la historia de nuestra especialidad, sino también representa una forma de proyección de una las variables relevantes al momento de abordar el déficit de

Tabla 1. Programas de formación de anestesiología 2014

\begin{tabular}{|lccc|}
\hline Universidad & Año de inicio & Acreditación & Ciudad \\
Universidad de Chile & 1965 & No acreditada & Santiago \\
Pontificia Universidad Católica de Chile & 1971 & Acreditada & Santiago \\
Universidad de Concepción & 1963 & No acreditada & Concepción \\
Universidad de Valparaíso & 1988 & Acreditada & Valparaíso \\
Universidad de Santiago & 1999 & Acreditada & Santiago \\
Universidad Mayor & 2005 & No acreditada & Santiago \\
Universidad del Desarrollo & 2009 & Acreditada & Santiago \\
Universidad de los Andes & 2009 & Acreditada & Santiago \\
Universidad Diego Portales & 2011 & No acreditada & Santiago \\
Universidad de la Frontera & 2011 & Acreditada & Temuco \\
Universidad Austral de Chile & 2013 & Acreditada & Valdivia \\
Universidad San Sebastián & 2013 & No Acreditada & Puerto Montt \\
Universidad Católica del Maule & 2013 & No Acreditada & Talca \\
Universidad Católica de la Santísima Concepción & 2014 & No Acreditada & Chillán \\
Universidad Andrés Bello & 2014 & No Acreditada & Talcahuano \\
Servicio de Salud Metropolitano Sur-Oriente & 2014 & No Acreditado & Santiago \\
\hline
\end{tabular}


anestesiólogos en el país: la formación de nuevos especialistas.

Los dos elementos básicos a considerar dentro de la formación de especialistas son la cantidad y la calidad con que ellos se forman y ambos elementos se encuentran entrañablemente ligados e influenciados por el plan de desarrollo que la universidad en cuestión hace previo y durante su desarrollo. Justamente para eso es que existe una agencia acreditadora, que toma en cuenta una serie de variables, al momento de decidir la posible acreditación y el período en que esta se encuentre vigente.

Dicho proceso de acreditación considera tantos elementos para determinar la calidad de un programa, que sería pretencioso que este artículo intentara abordar dicho tema. Sin perjuicio de ello, dentro de los datos aquí mencionados ustedes podrán identificar algunos que pudieran dar señales al respecto $y$ que dejo a su juicio.

\section{EL INICIO Y EL PROGRESO DE LOS PROGRAMAS DE FORMACIÓN DE ESPECIALISTAS}

Es difícil identificar el momento exacto en que la formación de anestesiólogos en Chile pasó a sustentarse en programas académicos estructurados, con duraciones definidas y con una plana docente claramente identificable. Esto puesto que por cierto, previo a dicho momento existía docencia y formación en anestesia, la cual se sustentaba en la calidad clínica e ímpetu docente de grandes anestesiólogos del país, muchos de los cuales hoy ostentan el reconocimiento de "Maestros de la Anestesiología Chilena".

Por tanto es probable que al menos en algunos casos, la metamorfosis de esas formaciones a lo que son hoy en día se produjera en forma progresiva en el tiempo, sin un punto de inicio preciso. En consecuencia, la única forma de buscar el "inicio oficial" de una de esas antiguas becas de anestesiología es recurrir a los propios centros formadores y solicitar los listados disponibles.

De acuerdo a esos listados, la beca de la Universidad de Concepción se inicia en el año 1963 con la formación de los Drs. Patricio Lagos y Guillermo Rodríguez; la beca de la Universidad de Chile se inicia el año 1965 con los Drs. Luis Bertoló y Margarita Polanco y la beca de la Universidad Católica se inicia el año 1971 con los Drs. Héctor Lacassie Silva, José de la Fuente y Stella Maettig. En forma muy posterior, en el año 1988, se inicia la beca de la Universidad de Valparaíso, cuando se constituye su Departamento de Anestesiología.
Sin embargo, las listas adolecen de información imprecisa, como lo demuestra el que por ejemplo, la Dra. Stella Maettig aparezca dentro de la nómina de becados de la Universidad de Chile en el período 1969 - 1972, además de egresada de la Universidad Católica el año 1974. Otro ejemplo: dentro del listado de la Universidad de Chile, entre los años 1976 y 1982 aparecen mencionados varios becados que coinciden con la nómina de la Universidad de Concepción, en cuyos casos el nombre aparece claramente vinculado con el Hospital Regional de Concepción, haciendo parecer que eran becados de la Universidad de Chile, pero con sede en dicho hospital.

Consultada la Dra. Maettig al respecto, señala que ella fue becada del SNS (Servicio Nacional de Salud) en la Universidad Católica, desarrollando una parte importante de su beca en el Hospital Sótero del Río. Además, nos cuenta que la organización de la beca, la malla curricular y el acceso a la bibliografía existente en ese momento eran mucho más rudimentarios que los existentes hoy en día, por razones obvias. Cuando se le consulta a qué atribuye ella el aparecer también en el listado de la Universidad de Chile, ella explica que eso se debe a que en esos años la Universidad de Chile tenía Escuela de Graduados, a diferencia de la Universidad Católica, por lo que quien llevaba el registro de los nuevos especialistas era la Universidad de Chile.

Es muy probable que esa misma sea la causa por la cual la nómina de la Universidad de Chile también tenga incluidos becados de la Universidad de Concepción.

En todo caso, ello da cuenta de un proceso formativo y de registro incipientes en el país. Siendo esas las condiciones de aquel momento y consciente de que mi decisión significa replicar nombres, las nóminas publicadas en este artículo no se encuentran modificadas y son en consecuencia una copia fiel de lo que fue enviado por cada universidad.

Como lo muestra la Tabla 2, hasta principios de la década del 2000, la formación de anestesiólogos fue sustentada principalmente en el funcionamiento de 5 programas. Cuatro de estos eran programas universitarios y se encontraban acreditados ante la entidad que en esos tiempos ejercía esta labor: ASOFAMECH. Esos programas eran el de la Universidad de Chile, Pontifica Universidad Católica de Chile, Universidad de Concepción y Universidad de Valparaíso.

A fines de la década del 80 la suma de estos 4 programas no aportaba al medio más de una docena de nuevos especialistas, en circunstancias que el déficit de ellos era tanto o más dramático que el existente en la actualidad. 
Tabla 2. Número de egresados por año y por programa universitario

\begin{tabular}{|c|c|c|c|c|c|c|c|c|c|c|}
\hline Egreso & UCH & PUC & UDC & UDV & USACH & UM & UDD & UDLA & UDP & UFRO \\
\hline 1965 & & & 2 & & & & & & & \\
\hline 1968 & 2 & & & & & & & & & \\
\hline 1969 & 1 & & & & & & & & & \\
\hline 1970 & 1 & & & & & & & & & \\
\hline 1971 & 1 & & & & & & & & & \\
\hline 1972 & 3 & & 1 & & & & & & & \\
\hline 1973 & 1 & & & & & & & & & \\
\hline 1974 & 4 & 3 & & & & & & & & \\
\hline 1975 & & 2 & 1 & & & & & & & \\
\hline 1976 & 2 & & 1 & & & & & & & \\
\hline 1977 & 1 & 1 & 1 & & & & & & & \\
\hline 1978 & 5 & & & & & & & & & \\
\hline 1979 & 5 & 1 & 1 & & & & & & & \\
\hline 1980 & 12 & & 3 & & & & & & & \\
\hline 1981 & 8 & 3 & 1 & & & & & & & \\
\hline 1982 & 9 & 4 & 2 & & & & & & & \\
\hline 1983 & 5 & 6 & 4 & & 1 & & & & & \\
\hline 1984 & 4 & 2 & 1 & & & & & & & \\
\hline 1985 & 7 & 3 & & & & & & & & \\
\hline 1986 & 5 & 6 & 1 & & & & & & & \\
\hline 1987 & 8 & 3 & 1 & & 2 & & & & & \\
\hline 1988 & & 4 & & & & & & & & \\
\hline 1989 & 3 & 5 & 1 & & & & & & & \\
\hline 1990 & 6 & 5 & 1 & & 3 & & & & & \\
\hline 1991 & 5 & 5 & & 2 & 3 & & & & & \\
\hline 1992 & 3 & 5 & 3 & 2 & 5 & & & & & \\
\hline 1993 & 5 & 6 & 3 & 2 & 4 & & & & & \\
\hline 1994 & 3 & 7 & 2 & 1 & 3 & & & & & \\
\hline 1995 & 6 & 7 & 2 & 3 & 5 & & & & & \\
\hline 1996 & 5 & 7 & 2 & 4 & 24 & & & & & \\
\hline 1997 & 7 & 8 & 4 & 10 & 3 & & & & & \\
\hline 1998 & 7 & 11 & 3 & 5 & 8 & & & & & \\
\hline 1999 & 6 & 10 & 3 & 4 & 8 & & & & & \\
\hline 2000 & 7 & 8 & 2 & 7 & 5 & & & & & \\
\hline 2001 & 8 & 9 & 4 & 4 & 6 & & & & & \\
\hline 2002 & 9 & 9 & 2 & 4 & 6 & & & & & \\
\hline 2003 & 10 & 10 & 3 & 2 & 6 & & & & & \\
\hline 2004 & 8 & 10 & 3 & 5 & 5 & & & & & \\
\hline 2005 & 6 & 11 & 2 & 5 & 3 & & & & & \\
\hline 2006 & 8 & 11 & 3 & 2 & 2 & & & & & \\
\hline 2007 & 11 & 11 & 2 & 3 & 3 & & & & & \\
\hline 2008 & 11 & 11 & 3 & 4 & 3 & 2 & & & & \\
\hline 2009 & 10 & 11 & 3 & 4 & 4 & 3 & & & & \\
\hline 2010 & 7 & 10 & 3 & 3 & 5 & 3 & & & & \\
\hline 2011 & 10 & 11 & 2 & 4 & 4 & 6 & & & & \\
\hline 2012 & 12 & 10 & 5 & 7 & 7 & 3 & 2 & 3 & & \\
\hline 2013 & 12 & 10 & 5 & 3 & 7 & 3 & 2 & 3 & & \\
\hline 2014 & 11 & 14 & 5 & 5 & 8 & 2 & 2 & 3 & 1 & 1 \\
\hline Total & 280 & 270 & 91 & 95 & 143 & 22 & 6 & 9 & 1 & 1 \\
\hline
\end{tabular}

UCH-Universidad de Chile; PUC-Universidad Católica; UDC-Universidad de Concepción; UDV-Universidad de Valparaíso; USACH-Universidad de Santiago; UM-Universidad Mayor; UDD-Universidad del Desarrollo; UDLAUniversidad de los Andes; UDP-Universidad Diego Portales; UFRO-Universidad de la Frontera 
Producto de ello es que diferentes hospitales, agobiados por la falta de anestesiólogos, iniciaron planes de formación extra universitarios en que sus servicios organizaban becas, utilizando además como campo clínico los servicios de anestesia de los institutos de referencia nacional, cuyos egresados debían posteriormente rendir el examen de CONACEM, para obtener la certificación correspondiente.

Es así como tuvieron programas de este tipo hospitales como El Salvador, San Borja Arriarán, Roberto del Río y Posta Central, contando con la colaboración de hospitales como el Instituto de Neurocirugía, el Instituto Nacional del Tórax, el Hospital Luis Calvo Mackenna, el Hospital Caupolicán Pardo Correa y otros.

Los líderes de uno de estos programas, el de la Posta Central, sostuvieron conversaciones con el Ministerio de Salud durante el año 1993, con el fin de reunir a la gran mayoría de estos programas extra oficiales en uno solo. Derivado de esas conversaciones, ese año se constituyó el Programa Ministerial de Formación de Especialistas en Anestesiología, con sede en la Posta Central (actual Hospital de Urgencia Asistencia Pública) y con participación conjunta de varios de los hospitales e institutos mencionados previamente.

Posterior a ello, las autoridades de dicho programa sostuvieron conversaciones con el Presidente de la Sociedad de Anestesiología de Chile de aquel entonces, Dr. Guillermo Lema, concordando en que la Sociedad reconociera el funcionamiento de esta beca, dejando establecido que los egresados de ella debían rendir el examen de CONACEM una vez que completaran 5 años de ejercicio, incluido el tiempo destinado a la beca.

De este modo, surge el quinto programa de formación en esos años, el cual funciona exclusivamente como Programa Ministerial hasta el año 1999, en que la Universidad de Santiago negocia la posibilidad de hacer de dicho programa - ya con más de diez años de experiencia - su Programa de Post Título en Anestesiología. A partir de ese momento inician su ingreso becados elegidos a través del concurso de dicha Universidad.

Como se puede observar en la Tabla 2, de los 919 especialistas egresados hasta el año 2014, 280 lo hacen de la Universidad de Chile, 270 de la Pontificia Universidad Católica de Chile, 91 de la Universidad de Concepción, 95 de la Universidad de Valparaíso y 143 del Programa Ministerial-Universidad de Santiago de Chile, considerado este último como un solo programa. La sumatoria de todos ellos aporta 879 especialistas al país.

A partir del año 2005 se incorporan sucesivamente once programas nuevos, de los cuales cinco ya tienen egresados y seis aún no. La gran mayoría de ellos ha optado por un inicio progresivo con promociones iniciales de pocos becados, en busca de un proceso de consolidación docente ordenado. Sin embargo uno de ellos, el de la Universidad Diego Portales, optó por realizar un aumento brusco de tres cupos en su primera promoción a dieciocho en la segunda (aún en formación), cifra que se mantiene en rangos levemente inferiores en las dos promociones siguientes.

El aumento en la capacidad formadora de los antiguos programas más la suma de los nuevos, configura una progresión de la cifra de especialistas egresados por año o en formación, que se resume en el Gráfico 1. De acuerdo a este gráfico, entre los años 2015 y 2017 egresarán de estos programas 256 nuevos anestesistas. La Tabla 3 resume el aporte que hace cada programa a esta cifra global.

\section{CARACTERÍSTICAS PRINCIPALES DE CADA PROGRAMA}

Analizar cada programa en forma somera, persigue conocer sus características, campo clínico base,

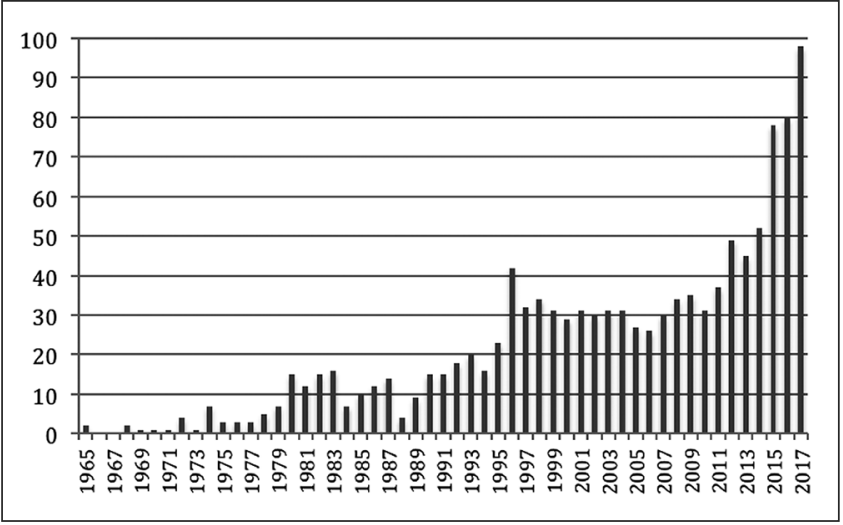

Gráfico 1. Sumatoria de los egresados anuales provenientes de todos los programas de formación. Las cifras de los años 1965 a 2014 corresponden a especialistas efectivamente egresados; las cifras de los años 2015 a 2017 corresponden a especialistas en formación. 
Tabla 3. Especialistas en formación entre los años 2014 y 2017. Contribución de cada programa en funcionamiento a la formación

\begin{tabular}{|c|c|c|c|c|}
\hline \multirow{2}{*}{ Programa } & \multicolumn{2}{|c|}{ Período } & \multicolumn{2}{|c|}{ Total } \\
\hline & $2012-2015$ & $2013-2016$ & $2014-2017$ & \\
\hline Universidad de Chile & 12 & 12 & 15 & 39 \\
\hline Pontificia Universidad Católica de Chile & 17 & 17 & 17 & 51 \\
\hline Universidad de Concepción & 6 & 6 & 7 & 19 \\
\hline Universidad de Valparaíso & 5 & 5 & 7 & 17 \\
\hline Universidad de Santiago & 8 & 7 & 8 & 23 \\
\hline Universidad Mayor & 3 & 2 & 4 & 9 \\
\hline Universidad del Desarrollo & 2 & 3 & 2 & 7 \\
\hline Universidad de los Andes & 5 & 7 & 9 & 21 \\
\hline Universidad Diego Portales & 18 & 12 & 11 & 41 \\
\hline Universidad de la Frontera & 2 & 2 & 3 & 7 \\
\hline Universidad Austral & & 2 & 3 & 5 \\
\hline Universidad San Sebastián & & 3 & 2 & 5 \\
\hline Universidad Católica del Maule & & 2 & 2 & 4 \\
\hline Universidad C de la Santísima Concepción & & & 3 & 3 \\
\hline Universidad Andrés Bello & & & 3 & 3 \\
\hline S. de Salud Metropolitano Sur Oriente & & & 2 & 2 \\
\hline Becados totales por promoción & 78 & 80 & 98 & 256 \\
\hline
\end{tabular}

campos clínicos principales, identificación de la jefatura, progresión del crecimiento en el número de becados y eventual condición de acreditación.

Además, se incorpora el listado de egresados por promoción, ya que este constituye un dato histórico. En estos listados ustedes podrán encontrar los nombres de sus compañeros de beca y también de quienes fueron sus docentes. Vuelvo a advertir que dado que la nómina se basa en los datos entregados por cada universidad, puede que existan errores $\mathrm{u}$ omisiones, que desde luego son involuntarias.

Cabe señalar que de acuerdo a los Requisitos Específicos para un Programa de Formación de Especialistas en Anestesiología, que tiene en cuenta la agencia acreditadora APICE en sus procesos de evaluación, la malla curricular debe contar a lo menos con estas rotaciones y duraciones respectivas:

- Anestesia en pabellón general 12 meses

- Anestesia neuroquirúrgica 2 meses

- Anestesia obstétrica 2 meses

- Anestesia pediátrica 3 meses

- Anestesia en cirugía

cardiovascular

3 meses

- Anestesia en cirugía de tórax 2 meses

- Anestesia en traumatología 2 meses

- Unidad de cuidados intensivos e intermedios

- Unidad de dolor

4-6 meses

- Turnos de urgencia o residencia Según rotación
En estos momentos es meridianamente claro que las rotaciones "cuello de botella" en la formación de especialistas en anestesiología, que producen restricción por encontrarse sobredemandadas respecto a la disponibilidad de campos clínicos son: anestesia pediátrica (sobretodo acceso a manejo de neonatos), anestesia neuroquirúrgica, anestesia para cirugía cardiovascular y anestesia para cirugía de tórax.

En consecuencia, este breve análisis de cada programa inquiere además en qué campo clínico están resolviendo el acceso de sus becados a estas rotaciones.

\section{UNIVERSIDAD DE CHILE}

Jefe de Programa: Dra. María Mercedes Aguirre Acreditación: El programa se presentó recientemente a acreditación ante APICE y se encuentra en estos momentos a la espera de que sea designada la Comisión de Pares Evaluadores.

Becados en tercer año: 12

Becados en segundo año: 12

Becados en primer año: 15

La Tabla 4 es la nómina de todos los egresados del Programa de Anestesiología de la Universidad de Chile desde el año 1968 hasta el año 2014. 
Tabla 4. Nomina de egresados del programa de anestesiología de la Universidad de Chile 1968 - 2014

\begin{tabular}{|c|c|c|}
\hline 1968 & $\begin{array}{l}\text { Luis Bertoló } \\
\text { Margarita Polanco }\end{array}$ & $\begin{array}{l}\text { JJ Aguirre } \\
\text { JJ Aguirre }\end{array}$ \\
\hline 1969 & Guillermo Ragni & JJ Aguirre \\
\hline 1970 & M. Eugenia Escobar & JJ Aguirre \\
\hline 1971 & Ivonne Guzmán & U Católica \\
\hline 1972 & $\begin{array}{l}\text { M. Patricia Vargas } \\
\text { Enrique Sepúlveda } \\
\text { Stella Maettig }\end{array}$ & $\begin{array}{l}\text { Deformes Valpo } \\
\text { Sótero del Río } \\
\text { Sótero del Río }\end{array}$ \\
\hline 1973 & Jorge Correa & JJ Aguirre \\
\hline 1974 & $\begin{array}{l}\text { Fernando Bravo } \\
\text { Vicente Hernández } \\
\text { Keske Matsumoto } \\
\text { M. del Carmen Bastidas }\end{array}$ & $\begin{array}{l}\text { Salvador } \\
\text { JJ Aguirre } \\
\text { Salvador } \\
\text { Salvador }\end{array}$ \\
\hline 1976 & $\begin{array}{l}\text { Oriana Maldonado } \\
\text { Ramón Danitz }\end{array}$ & $\begin{array}{l}\text { H. Concepción } \\
\text { P. Jaraquemada }\end{array}$ \\
\hline 1977 & Pilar Macho & Salvador \\
\hline 1978 & $\begin{array}{l}\text { Elba Bedoya } \\
\text { Raúl Venegas } \\
\text { Carmen Valenzuela } \\
\text { Lorenzo Fernández } \\
\text { Edward Rabah }\end{array}$ & $\begin{array}{l}\text { Salvador } \\
\text { P. Jaraquemada } \\
\text { S. Juan de Dios } \\
\text { H. Concepción } \\
\text { Salvador }\end{array}$ \\
\hline 1979 & $\begin{array}{l}\text { Samuel Barros } \\
\text { Jenny Rubina } \\
\text { Jorge Torregrosa } \\
\text { Fernanda Baeza } \\
\text { Gabriela Montt }\end{array}$ & $\begin{array}{l}\text { Salvador } \\
\text { P.Jaraquemada } \\
\text { S. Juan de Dios } \\
\text { JJ Aguirre } \\
\text { Salvador }\end{array}$ \\
\hline 1980 & $\begin{array}{l}\text { Sergio Rojas } \\
\text { Michael Zamberk } \\
\text { Carlos Riquelme } \\
\text { Aracelis Amadori } \\
\text { Julio Burotto } \\
\text { Bernardo Lilayu } \\
\text { Sara Maccioni } \\
\text { Óscar Retamal } \\
\text { M. Antonieta Rico } \\
\text { Marta Zúñiga } \\
\text { Cármen Águila } \\
\text { Margarita Rojas }\end{array}$ & $\begin{array}{l}\text { Salvador } \\
\text { P. Jaraquemada } \\
\text { S. Juan de Dios } \\
\text { Salvador } \\
\text { Salvador } \\
\text { S. Juan de Dios } \\
\text { S. Juan de Dios } \\
\text { H Concepción } \\
\text { Salvador } \\
\text { S. Juan de Dios } \\
\text { JJ Aguirre } \\
\text { Salvador }\end{array}$ \\
\hline 1981 & $\begin{array}{l}\text { M. Eliana Eberhard } \\
\text { José Tamblay } \\
\text { Patricia Barlaro } \\
\text { Rosemarie Kroneberg } \\
\text { Carlos Miranda } \\
\text { M. Cecilia Peña } \\
\text { Rubén Torres } \\
\text { Sara Montecinos }\end{array}$ & $\begin{array}{l}\text { S. Juan de Dios } \\
\text { JJ Aguirre } \\
\text { S. Juan de Dios } \\
\text { H. Concepción } \\
\text { H. Concepción } \\
\text { JJ Aguirre } \\
\text { H. Concepción } \\
\text { Salvador }\end{array}$ \\
\hline
\end{tabular}

\begin{tabular}{|c|c|c|}
\hline \multirow[t]{9}{*}{1982} & Wally Chávez & JJ Aguirre \\
\hline & Julio Guerra & Salvador \\
\hline & Iris Cox & Salvador \\
\hline & Juan de Toro & S. Juan de Dios \\
\hline & Marianne Herlitz & H. Concepción \\
\hline & Carlos Núñez & P. Jaraquemada \\
\hline & Juan Quiroz & Salvador \\
\hline & Ricardo Schurch & Salvador \\
\hline & Luis Brunet & JJ Aguirre \\
\hline \multirow[t]{5}{*}{1983} & Ruth Álvarez & Salvador \\
\hline & M. Inés Beddings & S. Juan de Dios \\
\hline & Guillermo Hidalgo & JJ Aguirre \\
\hline & Juan Pablo Ilic & Salvador \\
\hline & Patricio Barsocchini & JJ Aguirre \\
\hline \multirow[t]{4}{*}{1984} & Ricardo Cirio & S. Juan de Dios \\
\hline & M. Haydee Osses & JJ Aguirre \\
\hline & Ana María Rozas & S. Juan de Dios \\
\hline & Christian Toledo & JJ Aguirre \\
\hline \multirow[t]{7}{*}{1985} & Judith Billik & JJ Aguirre \\
\hline & Sergio Cerda & JJ Aguirre \\
\hline & Marcos Jara & P. Jaraquemada \\
\hline & Cristian Palma & P. Jaraquemada \\
\hline & Mario Poblete & JJ Aguirre \\
\hline & Raúl Sepúlveda & S. Juan de Dios \\
\hline & Anamaría Jacob & Salvador \\
\hline \multirow[t]{5}{*}{1986} & Nelson Cuadra & Salvador \\
\hline & M. Cristina Rondon & S. Juan de Dios \\
\hline & Norberto Bilbeny & JJ Aguirre \\
\hline & M. Silvana Cavallieri & Salvador \\
\hline & Alexandra Clericus & S. Juan de Dios \\
\hline \multirow[t]{8}{*}{1987} & Francisco Asenjo & JJ Aguirre \\
\hline & Patricia Bondi & JJ Aguirre \\
\hline & Jaime de la Maza & JJ Aguirre \\
\hline & Estela Maulén & JJ Aguirre \\
\hline & Jimena Rodríguez & JJ Aguirre \\
\hline & Carlos Tampier & JJ Aguirre \\
\hline & Roberto Zamorano & JJ Aguirre \\
\hline & Ïtalo Zamudio & JJ Aguirre \\
\hline \multirow[t]{3}{*}{1989} & Mario Contreras & JJ Aguirre \\
\hline & Marcela Labbe & JJ Aguirre \\
\hline & J. Alberto Muñoz & JJ Aguirre \\
\hline \multirow[t]{7}{*}{1990} & Erika Figueroa & JJ Aguirre \\
\hline & Carlos Aguayo & JJ Aguirre \\
\hline & M. Lucía Álvarez & JJ Aguirre \\
\hline & Lucía Barrientos & JJ Aguirre \\
\hline & J. José Galleguillos & JJ Aguirre \\
\hline & Juan Salgado & JJ Aguirre \\
\hline & Marco Guerrero & JJ Aguirre \\
\hline
\end{tabular}




\begin{tabular}{|c|c|c|}
\hline 1991 & $\begin{array}{l}\text { Javier Carvajal } \\
\text { Lorena Muñoz } \\
\text { Paul Sievers } \\
\text { Claudio Torres } \\
\text { Aurora Villablanca }\end{array}$ & $\begin{array}{l}\text { JJ Aguirre } \\
\text { JJ Aguirre } \\
\text { JJ Aguirre } \\
\text { JJ Aguirre } \\
\text { JJ Aguirre }\end{array}$ \\
\hline 1992 & $\begin{array}{l}\text { Mauricio González } \\
\text { Marco Olguín } \\
\text { Claudio Ramírez }\end{array}$ & $\begin{array}{l}\text { JJ Aguirre } \\
\text { JJ Aguirre } \\
\text { JJ Aguirre }\end{array}$ \\
\hline 1993 & $\begin{array}{l}\text { Luis Coronado } \\
\text { Roberto Peirano } \\
\text { M. Soledad Pérez } \\
\text { Miguel Santis } \\
\text { Carmen Silva }\end{array}$ & $\begin{array}{l}\text { J. Juan de Dios } \\
\text { JJ Aguirre } \\
\text { JJ Aguirre } \\
\text { JJ Aguirre } \\
\text { JJ Aguirre }\end{array}$ \\
\hline 1994 & $\begin{array}{l}\text { Andrés Stutzin } \\
\text { Carla Pellegrin } \\
\text { Alberto Laporte }\end{array}$ & $\begin{array}{l}\text { JJ Aguirre } \\
\text { Salvador } \\
\text { JJ Aguirre }\end{array}$ \\
\hline 1995 & $\begin{array}{l}\text { J. Pablo Acuña } \\
\text { Eugenia Torres } \\
\text { Verónica Varas } \\
\text { Roxana Lemus } \\
\text { Mariela Agurto } \\
\text { Orlando Alfaro }\end{array}$ & $\begin{array}{l}\text { JJ Aguirre } \\
\text { JJ Aguirre } \\
\text { JJ Aguirre } \\
\text { JJ Aguirre } \\
\text { S. Juan de Dios } \\
\text { S. Juan de Dios }\end{array}$ \\
\hline 1996 & $\begin{array}{l}\text { Mauricio Campos } \\
\text { Ricardo Cea } \\
\text { J. Carlos Muñoz } \\
\text { María Luz Rubio } \\
\text { Raúl Vega }\end{array}$ & $\begin{array}{l}\text { JJ Aguirre } \\
\text { JJ Aguirre } \\
\text { JJ Aguirre } \\
\text { JJ Aguirre } \\
\text { JJ Aguirre }\end{array}$ \\
\hline 1997 & $\begin{array}{l}\text { Víctor Parra } \\
\text { Tomás Pérez } \\
\text { Luz Mónica Poblete } \\
\text { Katherine Bancalari } \\
\text { Rodrigo Díaz G. } \\
\text { Ximena Mora } \\
\text { Marcela Sepúlveda }\end{array}$ & $\begin{array}{l}\text { JJ Aguirre } \\
\text { JJ Aguirre } \\
\text { JJ Aguirre } \\
\text { S. Juan de Dios } \\
\text { S. Juan de Dios } \\
\text { Salvador } \\
\text { Salvador }\end{array}$ \\
\hline 1998 & $\begin{array}{l}\text { Lilian Bonilla } \\
\text { Pedro Goles } \\
\text { Roberto Tapia } \\
\text { Germán Valenzuela } \\
\text { Omar Wistuba } \\
\text { Denize Arochas } \\
\text { Florence Gazabatt }\end{array}$ & $\begin{array}{l}\text { JJ Aguirre } \\
\text { JJ Aguirre } \\
\text { JJ Aguirre } \\
\text { JJ Aguirre } \\
\text { JJ Aguirre } \\
\text { S. Juan de Dios } \\
\text { S. Juan de Dios }\end{array}$ \\
\hline 1999 & $\begin{array}{l}\text { Carlos Alarcón } \\
\text { Cristian Arzola } \\
\text { Sandra Kunze } \\
\text { Ricardo Urtubia } \\
\text { José Guerra } \\
\text { Marco Jara }\end{array}$ & $\begin{array}{l}\text { JJ Aguirre } \\
\text { JJ Aguirre } \\
\text { JJ Aguirre } \\
\text { JJ Aguirre } \\
\text { S. Juan de Dios } \\
\text { S. Juan de Dios }\end{array}$ \\
\hline
\end{tabular}

\begin{tabular}{|c|c|c|}
\hline \multirow[t]{7}{*}{2000} & Álvaro Campos & JJ Aguirre \\
\hline & Gonzalo Campos & JJ Aguirre \\
\hline & Jaime Escobar & JJ Aguirre \\
\hline & Alejandro Recart & JJ Aguirre \\
\hline & Rodrigo Sepúlveda & JJ Aguirre \\
\hline & Alejandra Born & S. Juan de Dios \\
\hline & Ricardo Hernández & S. Juan de Dios \\
\hline \multirow[t]{8}{*}{2001} & M. Mercedes Aguirre & JJ Aguirre \\
\hline & Marcela Ávila & JJ Aguirre \\
\hline & Roberto Gálvez & JJ Aguirre \\
\hline & Patricio Mardones & JJ Aguirre \\
\hline & M. Eugenia Mellafe & JJ Aguirre \\
\hline & Loreto Muñoz & JJ Aguirre \\
\hline & Sergio Ramírez & JJ Aguirre \\
\hline & Marcelo Guzmán & S. Juan de Dios \\
\hline \multirow[t]{9}{*}{2002} & Ximena Campos & JJ Aguirre \\
\hline & Jaime Godoy & JJ Aguirre \\
\hline & Patricio Leyton & JJ Aguirre \\
\hline & Susan Mariño & JJ Aguirre \\
\hline & Gabriela Ramírez & JJ Aguirre \\
\hline & Tomislav Rajevic & JJ Aguirre \\
\hline & Paula Rodríguez & JJ Aguirre \\
\hline & Jaime Altamirano & S. Juan de Dios \\
\hline & Jaime Pino & S. Juan de Dios \\
\hline \multirow[t]{10}{*}{2003} & J. Pablo Álvarez & JJ Aguirre \\
\hline & Francisco Álvarez & JJ Aguirre \\
\hline & Paulina Lagos & JJ Aguirre \\
\hline & Andrés Leiva & JJ Aguirre \\
\hline & Rodrigo Morales & JJ Aguirre \\
\hline & M. Antonieta Ribal & JJ Aguirre \\
\hline & Pablo Sánchez & JJ Aguirre \\
\hline & Gian Paolo Volpato & JJ Aguirre \\
\hline & M. Cristina Allen & S. Juan de Dios \\
\hline & Lorena Lobo & S. Juan de Dios \\
\hline \multirow[t]{8}{*}{2004} & M. Soledad Letelier & JJ Aguirre \\
\hline & Carlos Muñoz & JJ Aguirre \\
\hline & Rogers Ortega & JJ Aguirre \\
\hline & Ricardo Pérez & JJ Aguirre \\
\hline & Crsitian Rocco & JJ Aguirre \\
\hline & Yolanda Urrejola & JJ Aguirre \\
\hline & Nelson Indo & S. Juan de Dios \\
\hline & Andrés Miranda & S. Juan de Dios \\
\hline \multirow[t]{6}{*}{2005} & Felipe Bates & JJ Aguirre \\
\hline & Andrea Kammerer & JJ Aguirre \\
\hline & Yelena Pegova & JJ Aguirre \\
\hline & Paulina Vallejos & JJ Aguirre \\
\hline & Isabel Basten & S. Juan de Dios \\
\hline & Andrea Torrejón & S. Juan de Dios \\
\hline
\end{tabular}




\begin{tabular}{|c|c|c|}
\hline \multirow[t]{8}{*}{2006} & Gonzalo Andrighetti & JJ Aguirre \\
\hline & Patricia Alarcón & JJ Aguirre \\
\hline & Cristián Aranguiz & JJ Aguirre \\
\hline & Carolina Carrillo & JJ Aguirre \\
\hline & Virginia Montecinos & JJ Aguirre \\
\hline & Alicia Olivares & JJ Aguirre \\
\hline & José Luis Molina & S. Juan de Dios \\
\hline & Rodolfo Zbinden & S. Juan de Dios \\
\hline \multirow[t]{11}{*}{2007} & Dayami Alonso & JJ Aguirre \\
\hline & Karen Borchert & JJ Aguirre \\
\hline & Pamela Cofré & JJ Aguirre \\
\hline & Andrés Montero & JJ Aguirre \\
\hline & Antonello Penna & JJ Aguirre \\
\hline & Macarena Reinero & JJ Aguirre \\
\hline & Gonzalo Rivera & JJ Aguirre \\
\hline & Patricia Róbinson & JJ Aguirre \\
\hline & Marcelo Solano & JJ Aguirre \\
\hline & Ana Paula Godoy & S. Juan de Dios \\
\hline & Julio López & S. Juan de Dios \\
\hline \multirow[t]{10}{*}{2008} & Sergio Alegría & JJ Aguirre \\
\hline & Ivar Mendizabal & JJ Aguirre \\
\hline & Mónica Morales & JJ Aguirre \\
\hline & Juan Retamal & JJ Aguirre \\
\hline & Claudia Robin & JJ Aguirre \\
\hline & Hugo Torrealba & JJ Aguirre \\
\hline & Claudia Valdés & JJ Aguirre \\
\hline & Milko Zott & JJ Aguirre \\
\hline & Alejandro Beltrán & S. Juan de Dios \\
\hline & Giancarlo Gandolfi & S. Juan de Dios \\
\hline \multirow[t]{10}{*}{2009} & Paula Berardi & JJ Aguirre \\
\hline & Lorena Cáceres & JJ Aguirre \\
\hline & Paola Guglielmi & JJ Aguirre \\
\hline & Sandra Lataste & JJ Aguirre \\
\hline & Jocelyn Maurer & JJ Aguirre \\
\hline & Alejandra Ostojic & JJ Aguirre \\
\hline & Mariana Storaker & JJ Aguirre \\
\hline & Karen Venegas & JJ Aguirre \\
\hline & Carola Guzmán & S. Juan de Dios \\
\hline & Carolina Riffo & S. Juan de Dios \\
\hline \multirow[t]{7}{*}{2010} & María José Cordero & JJ Aguirre \\
\hline & Isabel Galleguillos & JJ Aguirre \\
\hline & Kimlen Godoy & JJ Aguirre \\
\hline & Urano Figueroa & JJ Aguirre \\
\hline & Olivia Revollo & JJ Aguirre \\
\hline & Denisse Penjean & S. Juan de Dios \\
\hline & Marcelo Bernardín & S. Juan de Dios \\
\hline
\end{tabular}

\begin{tabular}{|c|c|c|}
\hline \multirow[t]{10}{*}{2011} & Sebastián Andrighetti & JJ Aguirre \\
\hline & Jorge Castro & JJ Aguirre \\
\hline & Sebastián Monsálvez & JJ Aguirre \\
\hline & Víctor Lobos & JJ Aguirre \\
\hline & Juan Ignacio Egaña & JJ Aguirre \\
\hline & María Paz Rivas & JJ Aguirre \\
\hline & Sebastián Cintolesi & JJ Aguirre \\
\hline & Julio Perey & JJ Aguirre \\
\hline & Mariela Sepñulveda & S. Juan de Dios \\
\hline & Paula Contreras & S. Juan de Dios \\
\hline \multirow[t]{12}{*}{2012} & Daniela Bravo & JJ Aguirre \\
\hline & Sebastián Mayanz & JJ Aguirre \\
\hline & Felipe Maldonado & JJ Aguirre \\
\hline & Matías Cáceres & JJ Aguirre \\
\hline & Sebastián Eulufi & JJ Aguirre \\
\hline & M. Carolina Leal & JJ Aguirre \\
\hline & Alejandra Serrá & JJ Aguirre \\
\hline & Pamela Silva & JJ Aguirre \\
\hline & Ana María Tartari & JJ Aguirre \\
\hline & Antonieta Pinilla & S. Juan de Dios \\
\hline & Fernando Zurita & S. Juan de Dios \\
\hline & Viviana Alam & S. Juan de Dios \\
\hline \multirow[t]{12}{*}{2013} & Juan Morales & JJ Aguirre \\
\hline & Víctor de la Torre & JJ Aguirre \\
\hline & Javiera rojas & JJ Aguirre \\
\hline & Tomás Stamm & JJ Aguirre \\
\hline & Dego Navarrete & JJ Aguirre \\
\hline & Javiera Vargas & JJ Aguirre \\
\hline & Raúl Torres & JJ Aguirre \\
\hline & Julián Aliste & JJ Aguirre \\
\hline & M. Soledad Ramírez & S. Juan de Dios \\
\hline & Claudio Fuhrer & S. Juan de Dios \\
\hline & Andrea Gutiérrez & Cl. Las Condes \\
\hline & Vanessa Silva & Cl. Las Condes \\
\hline \multirow[t]{11}{*}{2014} & Pablo Barragán & JJ Aguirre \\
\hline & Luis Cárdenas & JJ Aguirre \\
\hline & Felipe Contraras & JJ Aguirre \\
\hline & Ana María Donoso & JJ Aguirre \\
\hline & Víctor Figueroa & JJ Aguirre \\
\hline & Paula Fuenzalida & JJ Aguirre \\
\hline & Sirleny Meza & JJ Aguirre \\
\hline & Rodrigo Pizarro & JJ Aguirre \\
\hline & Denny Muñoz & S. Juan de Dios \\
\hline & Anabella Aguilera & S. Juan de Dios \\
\hline & Christopher Dempster & Cl. Las Condes \\
\hline
\end{tabular}


El Gráfico 2, muestra la progresión en el número de becados en formación anual a partir del año 1968.

En la historia de este programa, se identifican cuatro campos clínicos diferentes que en algún momento funcionaron en forma paralela: los hospitales José Joaquín Aguirre, San Juan de Dios, Salvador y Paula Jaraquemada (actualmente San Borja Arriarán).

Es así como en la nómina de egresados cada uno tiene vinculado un campo clínico de origen en alguno de estos hospitales. Sin embargo, también se identifican algunos becados que tienen vinculado un hospital distinto a estos, dentro de los cuales se encuentra el Hospital Regional de Concepción, el Hospital Sótero del Río y el Hospital Deformes de Valparaíso.

A partir del año 1986, el programa deja de utilizar el campo clínico del Hospital Paula Jaraquemada y a partir del año 1998 deja de utilizar el campo clínico del Hospital del Salvador, concentrándose solamente en los hospitales José Joaquín Aguirre y San Juan de Dios. Posteriormente, a partir del año 2010 incorpora a la Clínica Las Condes, como su tercer campo clínico.

En la actual malla curricular, los hospitales base (José Joaquín Aguirre, San Juan de Dios y Clínica Las Condes) desarrollan el ciclo de formación básico de sus respectivos becados, mientras el programa teórico, los talleres y las pasadas de especialidad son comunes para todos ellos.

Este programa, que en los últimos años había ofrecido en forma constante 12 cupos por promoción, los cuales estaban siendo destinados en forma casi exclusiva al MINSAL, decide en el año 2014 aumentar este número a 16 , de manera de ofrecer financiamiento del Hospital José Joaquín Aguirre a 4 residentes, como un mecanismo de formar especialistas para su propio campo clínico.
Esta ampliación de cupos, no produce problemas de acceso a las rotaciones de anestesia neuroquirúrgica ni cardiovascular, donde los campos clínicos propios son autosuficientes, pero si produce problemas en las rotaciones de anestesia pediátrica y anestesia para cirugía de tórax. En consecuencia, la jefatura del programa ha planificado incorporar un segundo campo clínico anexo al Hospital Luis Calvo Mackenna en anestesia pediátrica. Por su parte, la rotación en anestesia para cirugía de tórax incluye un mes en el Hospital José Joaquín Aguirre, un mes en el Instituto Nacional del Tórax y talleres en fantomas que les permiten a los becados la adquisición de las competencias necesarias.

\section{PONTIFICIA UNIVERSIDAD CATÓLICA DE CHILE}

Jefe del Programa: Dr. Alejandro Delfino

Acreditación: El programa se encuentra acreditado por APICE, según consta en el Acuerdo $\mathrm{N}^{\mathrm{o}} 17 \mathrm{de}$ dicha entidad, otorgándole un período de vigencia de 5 años, hasta el 18 de marzo de 2016.

Becados en tercer año: 17

Becados en segundo año: 17

Becados en primer año: 17

La Tabla 5 es la nómina de todos los egresados del Programa de Anestesiología de la Pontifica Universidad Católica de Chile desde el año 1974 hasta el año 2014.

El Gráfico 3, muestra la progresión en el número de becados en formación anual a partir del año 1974.

Este programa se desarrolla fundamentalmente en el Hospital Clínico de la Pontificia Universidad Católica de Chile y en el Hospital Sótero del Río, con el cual la Universidad sostiene un convenio

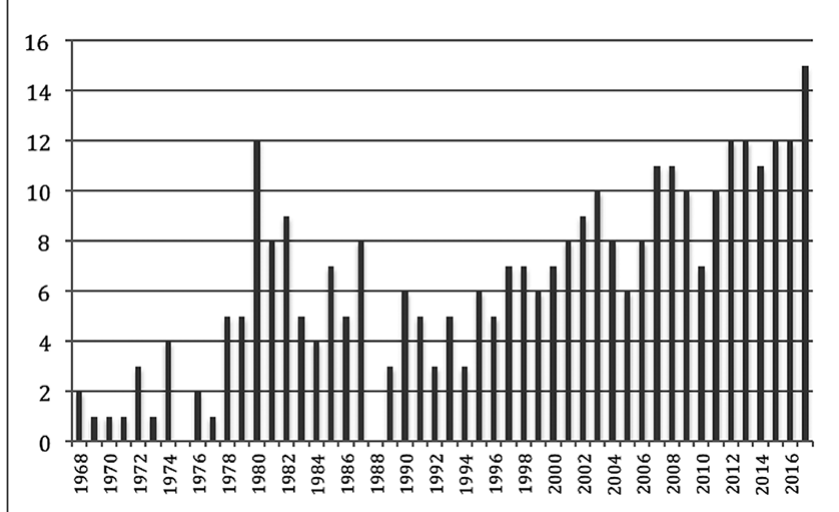

Gráfico 2. Becados formados anualmente en el Programa de Anestesiología de la Universidad de Chile 1968 - 2017 
Tabla 5. Nómina de egresados del programa de anestesiología de la Pontificia Universidad Católica de Chile 1974 - 2014

\begin{tabular}{|c|c|}
\hline 1974 & $\begin{array}{l}\text { Héctor Lacassie S. } \\
\text { Stella Maettig } \\
\text { José de la Fuente }\end{array}$ \\
\hline 1975 & $\begin{array}{l}\text { Jorge Atisha } \\
\text { Samuel Torregrosa }\end{array}$ \\
\hline 1977 & Jorge Dagnino \\
\hline 1979 & Ricardo Bustamante \\
\hline 1981 & $\begin{array}{l}\text { Amanda Marambio } \\
\text { Marta Quiroga } \\
\text { Sara Domínguez }\end{array}$ \\
\hline 1982 & $\begin{array}{l}\text { Leopoldo Avendaño } \\
\text { Gustavo Cortés } \\
\text { Guillermo Lema } \\
\text { Orlando Correa }\end{array}$ \\
\hline 1983 & $\begin{array}{l}\text { Emilio Santelices } \\
\text { Alejandro Escobar } \\
\text { Hernán Rodríguez } \\
\text { Héctor Vergara } \\
\text { M. Isabel Muñoz } \\
\text { M. Gabriela Rodríguez }\end{array}$ \\
\hline 1984 & $\begin{array}{l}\text { Marta Arriagada } \\
\text { M. Angélica Silva }\end{array}$ \\
\hline 1985 & $\begin{array}{l}\text { Roberto Canessa } \\
\text { Luis Castillo } \\
\text { Miguel Ramos }\end{array}$ \\
\hline 1986 & $\begin{array}{l}\text { David Castro } \\
\text { Mario Guerrero } \\
\text { Ana María Palacios } \\
\text { Ramón Coloma } \\
\text { Mario Concha } \\
\text { J. Carlos Devoto }\end{array}$ \\
\hline 1987 & $\begin{array}{l}\text { Fernando Alberdi } \\
\text { M. Soledad Covarrubias } \\
\text { Patricia Heisse }\end{array}$ \\
\hline 1988 & $\begin{array}{l}\text { Sergio Troncoso } \\
\text { Víctor Ortubia } \\
\text { Hernán Muñoz } \\
\text { Abel Pizarro }\end{array}$ \\
\hline 1989 & $\begin{array}{l}\text { M. Elena Folch } \\
\text { Guillermo Bugedo } \\
\text { César Cárcamo } \\
\text { Alberto González } \\
\text { Humberto Véliz }\end{array}$ \\
\hline 1990 & $\begin{array}{l}\text { Manuel Benavente } \\
\text { Patricia Panace } \\
\text { Carla Sacco } \\
\text { J. Carlos Terán } \\
\text { Víctor Troncoso }\end{array}$ \\
\hline
\end{tabular}

\begin{tabular}{|c|c|}
\hline 1991 & $\begin{array}{l}\text { Eugenio Canessa } \\
\text { Alejandro González } \\
\text { J. Antonio Merino } \\
\text { Claudio Ricke } \\
\text { Jacqueline Schlein }\end{array}$ \\
\hline 1992 & $\begin{array}{l}\text { Rodrigo Gazmuri } \\
\text { Ximena Gostín } \\
\text { Gonzalo Mutizabal } \\
\text { Jorge Rufs } \\
\text { Julio Valenzuela }\end{array}$ \\
\hline 1993 & $\begin{array}{l}\text { Carlos Andrade } \\
\text { Oscar Cherres } \\
\text { Julio González } \\
\text { Jéssica Medel } \\
\text { José Miguel Montes } \\
\text { Mariana Serra }\end{array}$ \\
\hline 1994 & $\begin{array}{l}\text { Juan Abarca } \\
\text { Carmen Aguilera } \\
\text { Lorna Castillo } \\
\text { Sergio Chiang } \\
\text { Paola Guerrero } \\
\text { Claudio Pinto } \\
\text { Juan Urra }\end{array}$ \\
\hline 1995 & $\begin{array}{l}\text { Ricardo Alegría } \\
\text { Carolina Asenjo } \\
\text { Daniel Becker } \\
\text { J. Carlos de la Cuadra } \\
\text { Ignacio Huenchullán } \\
\text { Richard Vergara } \\
\text { Gilberto Vigueras }\end{array}$ \\
\hline 1996 & $\begin{array}{l}\text { Rodrigo Añazco } \\
\text { Constanza Bastidas } \\
\text { Jaime Campusano } \\
\text { Rudy Garrido } \\
\text { Christian Nilo } \\
\text { Rafael Ortúzar } \\
\text { Claudia Sáez }\end{array}$ \\
\hline 1997 & $\begin{array}{l}\text { Ricardo Barla } \\
\text { Ignacio Cortínez } \\
\text { Óscar de la Jara } \\
\text { Rubén Gallardo } \\
\text { Fernando Lamarca } \\
\text { Sergio Latrach } \\
\text { Marcelo Llanca } \\
\text { Juan Saavedra }\end{array}$ \\
\hline 1998 & $\begin{array}{l}\text { Francia Arce } \\
\text { Hugo Becerra } \\
\text { Paulo Carrasco } \\
\text { Rolf Fleckenstein } \\
\text { Andrea Gil }\end{array}$ \\
\hline
\end{tabular}

\begin{tabular}{|c|c|}
\hline & $\begin{array}{l}\text { Héctor Lacassie Q. } \\
\text { Verónica Mertz } \\
\text { Rodolfo Molina } \\
\text { Lorena Ojeda } \\
\text { Arnaldo Pérez } \\
\text { Ivetlisse Solís }\end{array}$ \\
\hline 1999 & $\begin{array}{l}\text { Patricia Alarcón } \\
\text { Verena Brandes } \\
\text { Rodrigo Díaz C. } \\
\text { Bernardo Jorquera } \\
\text { Alfredo Llanos } \\
\text { Pedro Oliva } \\
\text { Claudia Sabat } \\
\text { Javier Torres } \\
\text { Claudio Vargas } \\
\text { Susan Veitl }\end{array}$ \\
\hline 2000 & $\begin{array}{l}\text { Carolina Beriswyil } \\
\text { Judith Bordones } \\
\text { Alejandro Bruhn } \\
\text { Magrit Calderón } \\
\text { Mario Cariaga } \\
\text { Jorge Gigoux } \\
\text { Rodrigo Rivas } \\
\text { Gonzalo Zúñiga }\end{array}$ \\
\hline 2001 & $\begin{array}{l}\text { Fernando Altermatt } \\
\text { Luis Azocar } \\
\text { Mauricio Ibacache } \\
\text { Rodrigo Montaña } \\
\text { Inés Órdenes } \\
\text { Teresita Santa María } \\
\text { Solange Saravia } \\
\text { Jorge Seguel } \\
\text { Maricarmen Viñuela }\end{array}$ \\
\hline 2002 & $\begin{array}{l}\text { Jorge Aguilera } \\
\text { Ingrid Baudoin } \\
\text { René de la Fuente } \\
\text { Rodrigo Lantadilla } \\
\text { Paula Meléndez } \\
\text { Gastón Núñez } \\
\text { Germán Seckel } \\
\text { David Torres } \\
\text { Miguel Vega }\end{array}$ \\
\hline 2003 & $\begin{array}{l}\text { Cristian Carrasco } \\
\text { Winston Carrasco } \\
\text { Jorge Contreras } \\
\text { Humaya Curi Curi } \\
\text { Claudio Fierro } \\
\text { Ricardo Fuentes } \\
\text { Lorena Sariego } \\
\text { Erika Tirapegui } \\
\text { Diego Villegas } \\
\text { Carlos Castillo }\end{array}$ \\
\hline
\end{tabular}




\begin{tabular}{|c|c|c|c|c|c|}
\hline 2004 & $\begin{array}{l}\text { Nicolás Aeischleman } \\
\text { Paulo Aravena } \\
\text { Aldo Campusano } \\
\text { Carolina Cardemil } \\
\text { Alejandro Delfino } \\
\text { Jaime Etchepare } \\
\text { Rodrigo Fernández } \\
\text { Manuel Gallardo } \\
\text { Constanza Larraguibel } \\
\text { Claudio Nazar }\end{array}$ & \multirow[t]{2}{*}{$\begin{array}{r}2007 \\
2008\end{array}$} & \multirow{2}{*}{$\begin{array}{l}\text { Gabriela Kusmanic } \\
\text { M. Soledad Lienlaf } \\
\text { Kristina Petersen } \\
\text { Mathieu Reveco } \\
\text { Jaime Troncoso } \\
\text { Rodrigo López } \\
\text { M. Esperanza Carrasco } \\
\text { Ximena Gómez } \\
\text { Magdalena Fernandois } \\
\text { Ana María Oliveros } \\
\text { J. Edgardo Flores } \\
\text { Rodrigo Muñoz } \\
\text { Laura Gebauer } \\
\text { Jaime Vargas } \\
\text { Rodrigo Flores } \\
\text { Daria Galdames }\end{array}$} & 2011 & $\begin{array}{l}\text { M. Teresa Donoso } \\
\text { Carolina Barría } \\
\text { M. Eduardo Bonacic } \\
\text { Julio Brousse } \\
\text { Jorge Flores } \\
\text { Nelson Fuentealba } \\
\text { Maite Fuentes } \\
\text { Macarena Gilbert } \\
\text { Ximena Jara } \\
\text { J. Cristobal Pedemonte } \\
\text { Giselle Symmes }\end{array}$ \\
\hline 2005 & $\begin{array}{l}\text { M. Ignacia Gazmuri } \\
\text { Paula León } \\
\text { Cristian Muller } \\
\text { Pablo Muñoz } \\
\text { Mónica Olave } \\
\text { Ricardo Sierra } \\
\text { Natalia Sologuren } \\
\text { Loreto Urrutia } \\
\text { Carola Urrutia } \\
\text { Paula Valdebenito } \\
\text { Claudio Villagra }\end{array}$ & & & 2012 & $\begin{array}{l}\text { Javiera Benavides } \\
\text { Francisca Cabrera } \\
\text { M. José Contardo } \\
\text { Edwin Coronel } \\
\text { Francisco Espinoza } \\
\text { Loreto Mosqueira } \\
\text { M. Paz Olhagaray } \\
\text { Liliana Ramos } \\
\text { M. Olivia Rondanelli } \\
\text { Carla Hudson }\end{array}$ \\
\hline \multirow[t]{2}{*}{2006} & $\begin{array}{l}\text { Darwin Acuña } \\
\text { Denis Arzabe } \\
\text { Loreto Bahamondes } \\
\text { Marco Balkenhol } \\
\text { M. Francisca Bernucci } \\
\text { Marcia Corvetto } \\
\text { Rose Marie Heider } \\
\text { Waldo Merino } \\
\text { Paulina Muñoz } \\
\text { Manuel Arriagada }\end{array}$ & 2010 & $\begin{array}{l}\text { Alvaro Elgueta } \\
\text { M. Francisca Elgueta } \\
\text { Andrea González } \\
\text { Verónica Lazo } \\
\text { Gonzalo Millán } \\
\text { Denise Thibaut } \\
\text { Carlos Gallardo } \\
\text { Pía Alejandra Bravo } \\
\text { Marcos Rattalino } \\
\text { Matías Yacsich }\end{array}$ & 2013 & $\begin{array}{l}\text { Daniel Vargas } \\
\text { Víctor Vargas } \\
\text { Jorge Egger } \\
\text { M. José Aedo } \\
\text { Ignacio Berríos } \\
\text { Stephanie Cabrera } \\
\text { Javier Enríquez } \\
\text { Macarena Monsalve } \\
\text { Duby Moreno } \\
\text { Marcela Rodríguez }\end{array}$ \\
\hline & Pedro Oyaneder & & Eduardo Vega & 2014 & Luis Álamos \\
\hline 2007 & $\begin{array}{l}\text { Rodrigo Acuña } \\
\text { Ghislaine Echeverría } \\
\text { Paola Fuentes } \\
\text { Daniela Fuenzalida } \\
\text { Gonzalo Giralt } \\
\text { Katia González }\end{array}$ & & $\begin{array}{l}\text { Valeria Pantoja } \\
\text { Natalia Pérez } \\
\text { Carolina Romero } \\
\text { Carolina Martínez } \\
\text { Patricio Vega } \\
\text { Camila Venegas }\end{array}$ & & $\begin{array}{l}\text { Paulina Díaz } \\
\text { Carolina Ramírez } \\
\text { Antonieta Valderrama } \\
\text { Claudia Hurtado } \\
\text { Ana María Veloso } \\
\text { Andrea Araneda }\end{array}$ \\
\hline
\end{tabular}

docente-asistencial de muy larga data. Además, recientemente la Universidad ha incorporado el campo clínico del nuevo Hospital de la Florida, perteneciente al mismo Servicio de Salud Metropolitano Sur Oriente.

Sin embargo, a partir de la ampliación del número de cupos anuales de 12 a 17, este programa ha debido recurrir a algunos institutos de referencia nacional, para complementar pasadas como anestesia neuroquirúrgica y en cirugía de tórax, por lo cual en la actualidad sus becados rotan un mes por el Instituto de Neurocirugía y un mes por el Instituto Nacional del Tórax.

\section{UNIVERSIDAD DE CONCEPCIÓN}

Jefe de Programa: Dr. Joel Marchant

Acreditación: El programa no se ha presentado a acreditación ante APICE, fundamentalmente debido al deterioro de la planta de pabellones del Hospital Guillermo Grant Benavente de Concepción, a raíz del terremoto de febrero de 2010 , lo cual impe- 


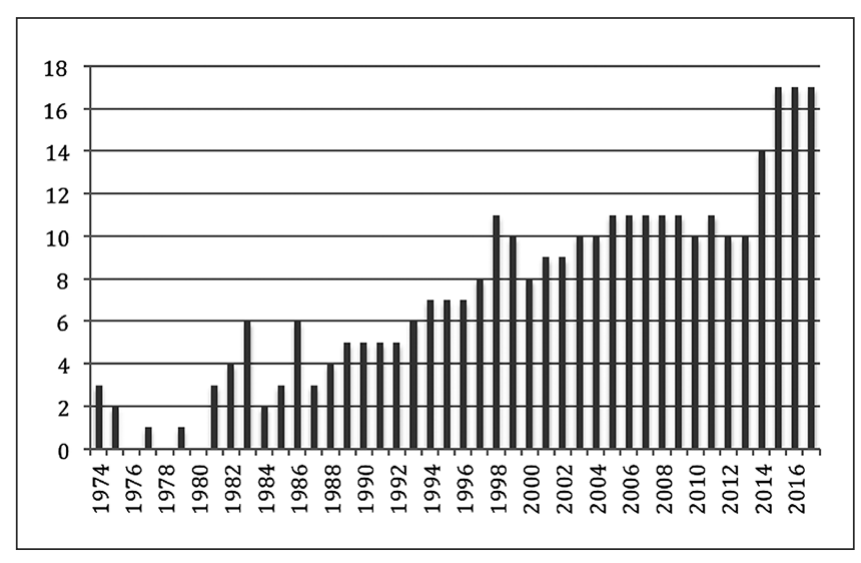

Gráfico 3. Becados formados anualmente en el Programa de Anestesiología de la Pontificia Universidad Católica de Chile 1974 - 2017. día a sus autoridades mostrar infraestructura acorde con la calidad del mismo. Las obras de reconstrucción de dicho establecimiento se encuentran $a d$ portas de ser entregadas, por lo cual probablemente en el corto plazo el programa iniciará este trámite

Becados en tercer año: 6

Becados en segundo año: 6

Becados en primer año: 7

La Tabla 6 es la nómina de todos los egresados del Programa de Anestesiología de la Universidad de Concepción desde el año 1965 hasta el año 2014.

El Gráfico 4, muestra la progresión en el número de becados en formación anual a partir del año 1965.

Este programa se desarrolla casi exclusivamente en el Hospital Guillermo Grant Benavente, donde según el Dr. Marchant existe la casuística suficiente en todas las rotaciones de la beca, incluidas las rotaciones por anestesia cardiovascular, pediátrica, neuroquirúrgica y en cirugía de tórax.

\section{UNIVERSIDAD DE VALPARAÍSO}

Jefe del Programa: Dra. Anamaría Correa Acreditación: El programa se encuentra acreditado por APICE, según consta en el Acuerdo $\mathrm{N}^{\circ} 67$ de dicha entidad, otorgándole un período de vigencia de 7 años, hasta el 26 de abril de 2020.

Becados en tercer año: 5

Becados en segundo año: 5

Becados en primer año: 7

La Tabla 7 es la nómina de todos los egresados del Programa de Anestesiología de la Universidad de Valparaíso desde el año 1991 hasta el año 2014.
El Gráfico 5, muestra la progresión en el número de becados en formación anual a partir del año 1991.

Este programa se desarrolla en varios hospitales de la quinta región, teniendo como sede principal a los hospitales Van Buren de Valparaíso, Gustavo Fricke de Viña del Mar y Naval Almirante Nef.

Si bien cuenta con una muy buena evaluación en su trámite de acreditación, siendo por el momento el programa que ha recibido la mayor cantidad de años (siete), sus autoridades han sido muy cautas en ofrecer un crecimiento del número de becas prudente, siempre teniendo en cuenta contar con campo clínico suficiente.

Es por esta razón, que en estos momentos este programa no tiene problemas de acceso a las rotaciones previamente señaladas como deficitarias, puesto que sus campos clínicos son suficientes en relación a los becados en formación.

\section{UNIVERSIDAD DE SANTIAGO}

Jefe del Programa: Dr. Víctor Hanna

Acreditación: El programa se encuentra acreditado por APICE, según consta en el Acuerdo $\mathrm{N}^{\mathrm{o}} 75 \mathrm{de}$ dicha entidad, otorgándole un período de vigencia de 3 años, hasta el 28 de junio de 2016.

Becados en tercer año: 8

Becados en segundo año: 7

Becados en primer año: 8

La Tabla 8 es la nómina de todos los egresados del Programa desde que este era Programa Ministerial hasta que se convirtió en el Programa de Anestesiología de la Universidad de Santiago, vale decir, desde el año 1983 hasta el año 2014. 
Tabla 6. Nómina de egesados del programa de anestesiología de la Universidad de Concepción 1965 -2014

\begin{tabular}{|c|c|}
\hline 1965 & $\begin{array}{l}\text { Patricio Lagos } \\
\text { Guillermo Rodríguez }\end{array}$ \\
\hline 1972 & Ester Marcuse \\
\hline 1975 & Luigi Andriani \\
\hline 1976 & Oriana Maldonado \\
\hline 1977 & Carlos Rojas \\
\hline 1977 & Lorenzo Fernández \\
\hline 1980 & $\begin{array}{l}\text { Óscar Retamal } \\
\text { Carlos Miranda } \\
\text { Rubén Torres }\end{array}$ \\
\hline 1981 & Rose Marie Kroneberg \\
\hline 1982 & $\begin{array}{l}\text { Marianne Herlitz } \\
\text { Ruth Pincheira }\end{array}$ \\
\hline 1983 & $\begin{array}{l}\text { Jéssica Israel } \\
\text { M. Eliana Irarrázaval } \\
\text { Ximena Castillo } \\
\text { Orietta Urrejola }\end{array}$ \\
\hline 1984 & Elizabeth Ross \\
\hline 1986 & Marcos González \\
\hline 1987 & Carmen Zambrano \\
\hline 1989 & Rogelio Parra \\
\hline 1990 & M. Antonieta Puga \\
\hline 1992 & $\begin{array}{l}\text { Pamela Daza } \\
\text { Joel Marchant } \\
\text { Rogelio Ureta }\end{array}$ \\
\hline 1993 & $\begin{array}{l}\text { Rafael Ruiz } \\
\text { Edwin Henríquez } \\
\text { Patricia Pezo }\end{array}$ \\
\hline 1994 & $\begin{array}{l}\text { Edgardo Bassaletti } \\
\text { Raúl Ortega }\end{array}$ \\
\hline
\end{tabular}

\begin{tabular}{|c|c|}
\hline 1995 & $\begin{array}{l}\text { Juana Herrera } \\
\text { Francisco Garrido }\end{array}$ \\
\hline 1996 & $\begin{array}{l}\text { Antonio Salinas } \\
\text { Guillermo Ramírez }\end{array}$ \\
\hline 1997 & $\begin{array}{l}\text { Francisco Muñoz } \\
\text { Edith Quintulén } \\
\text { Marisol Rey } \\
\text { José Toro }\end{array}$ \\
\hline 1998 & $\begin{array}{l}\text { José Luis Cuevas } \\
\text { Helia González } \\
\text { Guillermo Navarro }\end{array}$ \\
\hline 1999 & $\begin{array}{l}\text { M. Ángela Cuevas } \\
\text { Viviana Hernández } \\
\text { Jenny Montero }\end{array}$ \\
\hline 2000 & $\begin{array}{l}\text { Patricio Cárdenas } \\
\text { Óscar Peña }\end{array}$ \\
\hline 2001 & $\begin{array}{l}\text { Ingelore Follert } \\
\text { Cristian Lederman } \\
\text { Marianela Núñez } \\
\text { Paula Romero }\end{array}$ \\
\hline 2002 & $\begin{array}{l}\text { Crisóstomo Fonseca } \\
\text { Paulina Carbonell }\end{array}$ \\
\hline 2003 & $\begin{array}{l}\text { Julio Castro } \\
\text { Carolina Faúndez } \\
\text { John Valdebenito }\end{array}$ \\
\hline 2004 & $\begin{array}{l}\text { Renato Klenner } \\
\text { Fabiola Osorio } \\
\text { Hugo Soto }\end{array}$ \\
\hline 2005 & $\begin{array}{l}\text { Fernando Delgado } \\
\text { Octavio Martínez }\end{array}$ \\
\hline
\end{tabular}

\begin{tabular}{|c|c|}
\hline 2006 & $\begin{array}{l}\text { Felipe del Fierro } \\
\text { Eugenio Sanhueza } \\
\text { Pedro Fuentealba }\end{array}$ \\
\hline 2007 & $\begin{array}{l}\text { Juan Gálvez } \\
\text { Mariana Malte }\end{array}$ \\
\hline 2008 & $\begin{array}{l}\text { Gabriel Meza } \\
\text { Carlos Labrañan } \\
\text { Rodrigo Orrego }\end{array}$ \\
\hline 2009 & $\begin{array}{l}\text { Valeria Epulef } \\
\text { Nelson Pulgar } \\
\text { Erik Salazar }\end{array}$ \\
\hline 2010 & $\begin{array}{l}\text { Macarena Gallegos } \\
\text { Juan Alberto Lefiñir } \\
\text { Andrea Sánchez }\end{array}$ \\
\hline 2011 & $\begin{array}{l}\text { Vicente Bejar } \\
\text { Paula Carrasco }\end{array}$ \\
\hline 2012 & $\begin{array}{l}\text { Carlos Bolbarán } \\
\text { Ricardo Fuentealba } \\
\text { J. Carlos Barra } \\
\text { Diego Vergara } \\
\text { Bernardo Rodríguez }\end{array}$ \\
\hline 2013 & $\begin{array}{l}\text { Ketherine Dubos } \\
\text { Natalia Montero } \\
\text { Daniela Nieweld } \\
\text { Daniel Jiménez } \\
\text { Héctor Vidal }\end{array}$ \\
\hline 2014 & $\begin{array}{l}\text { Diego Maitre } \\
\text { Cesar Soto } \\
\text { Susana Ramírez } \\
\text { Óscar Meza } \\
\text { J. Pablo Medina }\end{array}$ \\
\hline
\end{tabular}

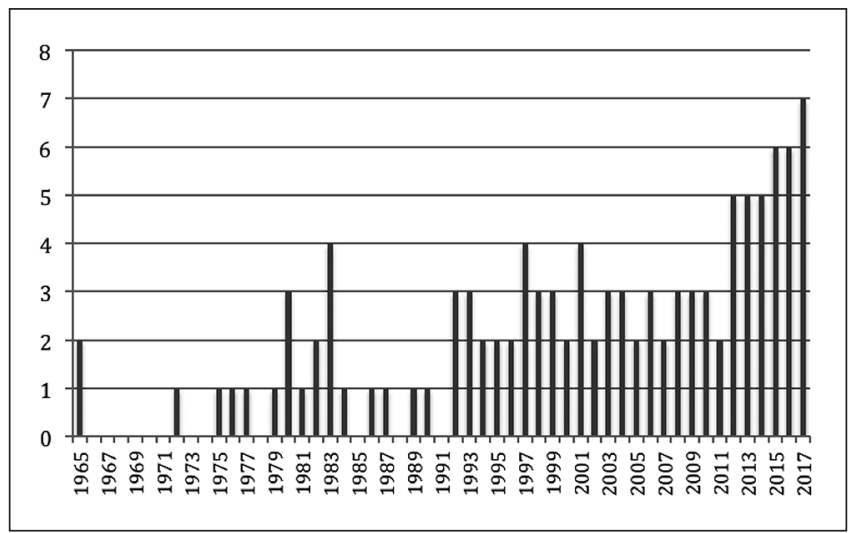

Gráfico 4. Becados formados anualmente en el Programa de Anestesiología de la Universidad de Concepción 1965 - 2017. 
Tabla 7. Nómina de egresados del programa de anestesiología de la Universidad de Valparaíso 1991 - 2014

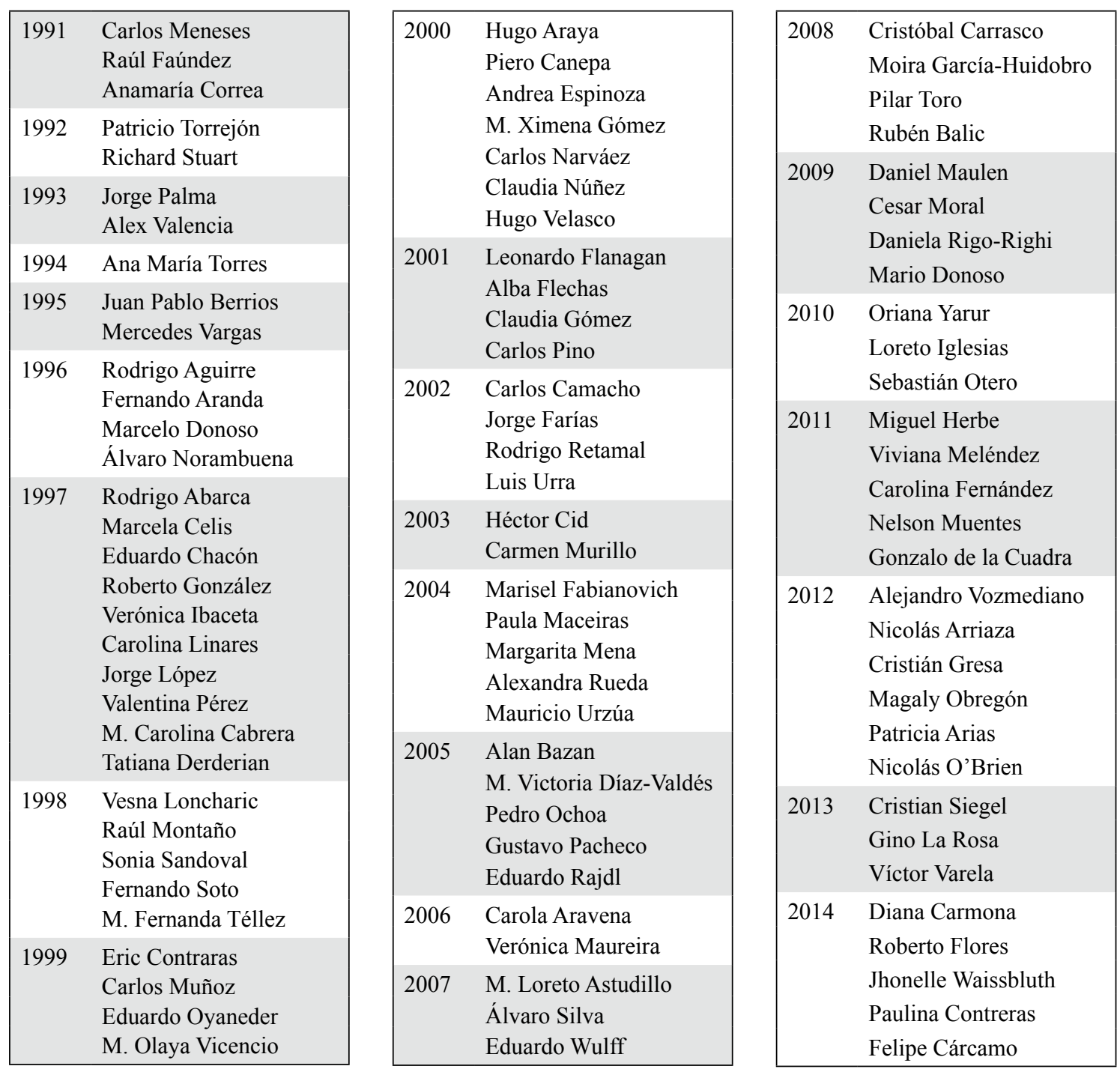

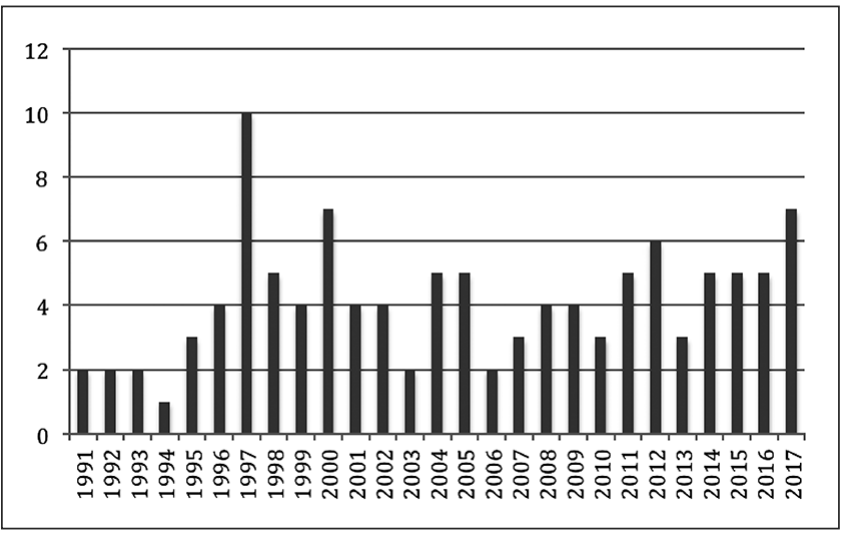

Gráfico 5. Becados formados anualmente en el Programa de Anestesiología de la Universidad de Valparaíso 1991 - 2017. 
Tabla 8. Nómina de egresados del programa ministerial Unoversidad de Santiago 1983 - 2014

\begin{tabular}{|c|c|}
\hline 1983 & Eliana Hidalgo \\
\hline 1987 & $\begin{array}{l}\text { Silvia Chicago } \\
\text { Mary Taiba }\end{array}$ \\
\hline 1990 & $\begin{array}{l}\text { Mauricio Ramos } \\
\text { Juan Rivera } \\
\text { Geisi Saito }\end{array}$ \\
\hline 1991 & $\begin{array}{l}\text { Enrique Moreno } \\
\text { Clara Luxoro } \\
\text { Renato Chacón }\end{array}$ \\
\hline 1992 & $\begin{array}{l}\text { Pamela Jorquera } \\
\text { Daniel Villalobos } \\
\text { Mauricio Jara } \\
\text { Henri Souyet } \\
\text { Suzan Macqueen }\end{array}$ \\
\hline 1993 & $\begin{array}{l}\text { Luis Belmar } \\
\text { Jaime Montiel } \\
\text { Mauricio Delgado } \\
\text { Héctor Ureta }\end{array}$ \\
\hline 1994 & $\begin{array}{l}\text { Gabriela Solari } \\
\text { Silvia Benedetto } \\
\text { Fernando Reyes }\end{array}$ \\
\hline 1995 & $\begin{array}{l}\text { Mini Fernández } \\
\text { Gonzalo de la Cuadra } \\
\text { Patricia Macaya } \\
\text { Marylen Oltra } \\
\text { Sandra Díaz }\end{array}$ \\
\hline 1996 & $\begin{array}{l}\text { Francisco Cadagan } \\
\text { Edgar Fernández } \\
\text { Andrea Moreno } \\
\text { José Guzmán } \\
\text { Melvin Sharp } \\
\text { Jorge Flores } \\
\text { Cecilia Aedo } \\
\text { Óscar Carrasco } \\
\text { Jaime Carrillo } \\
\text { Roberto Catalán } \\
\text { José Chávez } \\
\text { Guido Jelves } \\
\text { Wilma Méndez } \\
\text { Guillermo Molina } \\
\text { Roberto Moya } \\
\text { Ricardo Muñoz } \\
\text { Cecilia Perales } \\
\text { Daniel Ponce } \\
\text { Gema Sagredo } \\
\text { Yunny Vega } \\
\text { Sergio Avendaño } \\
\text { Cintia Montaño } \\
\text { Paola Ostermann } \\
\text { Alberto Velásquez }\end{array}$ \\
\hline
\end{tabular}

\begin{tabular}{|c|c|}
\hline 1997 & $\begin{array}{l}\text { Jaime Medina } \\
\text { Carmen Rojas } \\
\text { Mariana Varas }\end{array}$ \\
\hline 1998 & $\begin{array}{l}\text { Marco Báez } \\
\text { Claudia Campos } \\
\text { Mirko Guzmán } \\
\text { Michele Jaspard } \\
\text { Jaime Urbina } \\
\text { Héctor Maldonado } \\
\text { Germán Zegers } \\
\text { Patricia Aguayo }\end{array}$ \\
\hline 1999 & $\begin{array}{l}\text { René Cevo } \\
\text { M. Isabel Chávez } \\
\text { Pamela Graham } \\
\text { Gonzalo Mencía } \\
\text { José Meneses } \\
\text { Fernando Morales } \\
\text { Miguel Aichele } \\
\text { Giovana Camacho }\end{array}$ \\
\hline 2000 & $\begin{array}{l}\text { Marisol Castro } \\
\text { Arturo Vargas } \\
\text { Patricia Rojas } \\
\text { Fernando Moncayo } \\
\text { Rodrigo Durán }\end{array}$ \\
\hline 2001 & $\begin{array}{l}\text { Sandra Benavides } \\
\text { Axel Camousseight } \\
\text { Manuel Córdova } \\
\text { Paula Hasfura } \\
\text { Verónica Román } \\
\text { Carmen Yáñez }\end{array}$ \\
\hline 2002 & $\begin{array}{l}\text { Roberto Brito } \\
\text { Paulina Escandar } \\
\text { Olga Mora } \\
\text { Roberto Moreno } \\
\text { Cristian Rabb } \\
\text { Alicia Espinoza }\end{array}$ \\
\hline 2003 & $\begin{array}{l}\text { Rómulo Araya } \\
\text { Claudia Castro } \\
\text { Marcelo Concha } \\
\text { Carla Golppi } \\
\text { Lía Muñoz } \\
\text { Sebastián Sánchez }\end{array}$ \\
\hline 2004 & $\begin{array}{l}\text { Pablo González } \\
\text { Marisol Muñoz } \\
\text { Alejandro Orrego } \\
\text { M. Carolina Ramírez } \\
\text { Andrés Soto }\end{array}$ \\
\hline
\end{tabular}

\begin{tabular}{|c|c|}
\hline 2005 & $\begin{array}{l}\text { Miguel Ángel Núñez } \\
\text { Dennis Selma } \\
\text { Sasha Müller }\end{array}$ \\
\hline 2006 & $\begin{array}{l}\text { Héctor Ávila } \\
\text { Rodrigo Rodríguez }\end{array}$ \\
\hline 2007 & $\begin{array}{l}\text { Carlos Domínguez } \\
\text { Karime Herrera } \\
\text { Cristián Vera }\end{array}$ \\
\hline 2008 & $\begin{array}{l}\text { Arturo del Pino } \\
\text { Luciano González } \\
\text { Marco Ulloa }\end{array}$ \\
\hline 2009 & $\begin{array}{l}\text { Macarena Muñoz } \\
\text { M de lo Ángeles Oñate } \\
\text { Carla Quilodrán } \\
\text { Mauricio Zanni }\end{array}$ \\
\hline 2010 & $\begin{array}{l}\text { Benjamín Díaz } \\
\text { Paloma Domancic } \\
\text { César Madrid } \\
\text { Jaime Molina } \\
\text { Raúl Sepúlveda }\end{array}$ \\
\hline 2011 & $\begin{array}{l}\text { Magdalena Aristegui } \\
\text { Rosario Errázuriz } \\
\text { Francisco Elgueta } \\
\text { Omar Herrera }\end{array}$ \\
\hline 2012 & $\begin{array}{l}\text { Sandra Sommer } \\
\text { Sergio Quezada } \\
\text { Rodrigo Zamorano } \\
\text { Javiera Valeria } \\
\text { Gonzalo Meza } \\
\text { Etni Yáñez } \\
\text { Alejandra Peralta }\end{array}$ \\
\hline 2013 & $\begin{array}{l}\text { Daniela Zagal } \\
\text { Cristian Castillo } \\
\text { Manuel Cid } \\
\text { Diego Mora } \\
\text { Osvaldo Arriagada } \\
\text { Ximena Meza } \\
\text { Carlos López }\end{array}$ \\
\hline 2014 & $\begin{array}{l}\text { Rolando Mendoza } \\
\text { Stephanie García } \\
\text { Carlos Margarit } \\
\text { Héctor Valenzuela } \\
\text { Ïtalo Pesce } \\
\text { Sebastián Larraín } \\
\text { J. Pablo Maass } \\
\text { Pilar Martínez }\end{array}$ \\
\hline
\end{tabular}




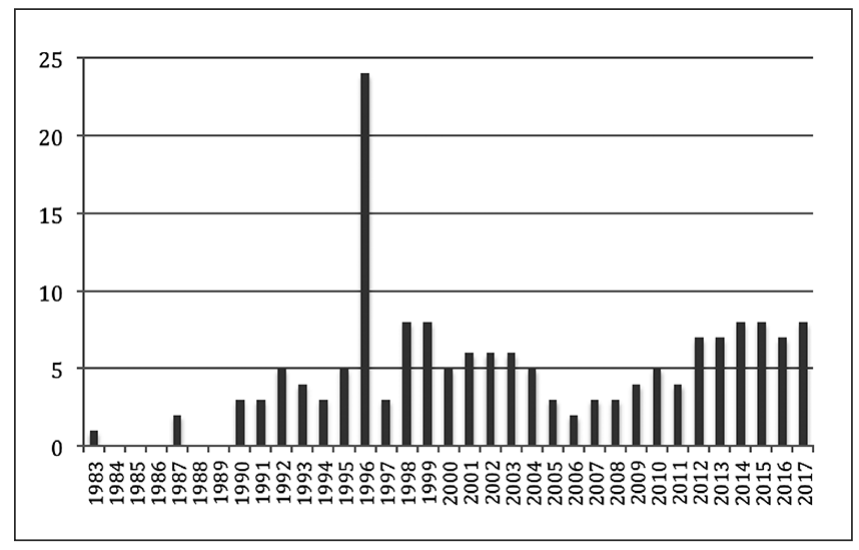

Gráfico 6. Becados formados anualmente en el Programa de Anestesiología Ministerial - Universidad de Santiago 1983 - 2017.
El Gráfico 6, muestra la progresión en el número de becados en formación anual a partir del año 1983.

En dicho gráfico ustedes notarán un peak de formación de becados que egresa el año 1996, período en el cual por presiones ministeriales, se recibió un gran número de residentes. Destaco esto, porque siendo parte de la plana docente que participó de dicho proceso, creo que haber cedido a tales presiones y aceptar tal número de becados fue un error importante, que este programa no ha vuelto a cometer.

Para refrendar esta impresión un solo dato: si se coteja dicho listado con los registros de CONACEM, esa es la promoción de esta beca en que porcentualmente menos egresados rindieron el examen de CONACEM, requisito que en aquel entonces era necesario para regularizar su certificación.

Este programa tiene sus campos clínicos base en el Hospital de Urgencia Asistencia Pública y en el Hospital San José y complementa sus rotaciones en los Institutos Nacional del Tórax y de Neurocirugía, y en los Hospitales Padre Hurtado y Luis Calvo Mackenna.

Durante muchos años, este programa fue el usuario casi exclusivo de campos clínicos como el Instituto Nacional del Tórax y el Instituto de Neurocirugía. De hecho, gran parte de los anestesiólogos de dichos institutos provienen de este programa. Sin embargo, hoy estos institutos son requeridos por varios otros de los programas de anestesiología, por lo cual la holgura que antes poseía este para utilizar dichos campos clínicos ha disminuido en función de un mejor aprovechamiento de la capacidad docente de esos servicios, tan necesaria para potenciar la formación general de anestesistas.

Es por esto, que el programa ha mantenido durante los últimos años su oferta de cupos en 8 anuales, limitado por la disponibilidad de dichas rotaciones, donde ocupa en forma pareja 2 plazas mensuales, lo cual determina su capacidad formadora máxima.

\section{UNIVERSIDAD MAYOR}

Jefe de Programa: Dr. Hernán Ramos

Acreditación: Según consta en la página web de APICE en el Acuerdo $\mathrm{N}^{0} 4$ de fecha 13 de agosto de 2010, el programa no fue acreditado luego de su evaluación.

Según se señala en la página web de la Universidad Mayor, esta habría apelado de la determinación de APICE ante la CNA (Comisión Nacional de Acreditación) y habría obtenido una acreditación de dos años, válida hasta el 9 de noviembre de 2013. Habiéndose cumplido dicha fecha, en la misma página web de la Universidad Mayor se señala "No acreditado por acreditación vencida".

Becados en tercer año: 3 Becados en segundo año: 2 Becados en primer año: 4

La Tabla 9 es la nómina de todos los egresados del Programa de Anestesiología de la Universidad Mayor, desde el año 2008 hasta el año 2014.

El Gráfico 7, muestra la progresión en el número de becados en formación anual a partir del año 2008.

El programa tiene su campo clínico base en el Hospital Dipreca y utiliza como campos clínicos asociados el Hospital Félix Bulnes y el Hospital de Carabineros.

La rotación de anestesia pediátrica la realizan en conjunto entre el Hospital de Carabineros y el Félix Bulnes, mientras las rotaciones de anestesia cardiovascular, neuroquirúrgica y en cirugía de tórax las realizan en el Hospital Dipreca. 
Tabla 9. Nómina de egresados del programa de anestesiología de la Universidad Mayor 2008 - 2014

\begin{tabular}{|c|c|c|c|c|c|}
\hline 2008 & $\begin{array}{l}\text { Andrés Monardes } \\
\text { Felipe Villarroel }\end{array}$ & \multirow[t]{7}{*}{2011} & \multirow{2}{*}{$\begin{array}{l}\text { Marcela Álvarez } \\
\text { Magdalena Vega }\end{array}$} & \multirow[t]{2}{*}{2012} & \multirow{2}{*}{$\begin{array}{l}\text { Paula Figueroa } \\
\text { Daniela Toledo } \\
\text { Jorge Darlic }\end{array}$} \\
\hline \multirow[t]{3}{*}{2009} & Manuel Durán & & & & \\
\hline & Beatriz Casanova & & \multirow{5}{*}{$\begin{array}{l}\text { Romiely Blanco } \\
\text { Alejandro Flores } \\
\text { Rodrigo Santander } \\
\text { Eugenia Clavero }\end{array}$} & \multirow[t]{3}{*}{2013} & Daniela Sepúlveda \\
\hline & Claudia Green & & & & Sebastián Rojas \\
\hline \multirow[t]{3}{*}{2010} & Francisca Casas & & & & Camilo Fernández \\
\hline & Jorge Miranda & & & 2014 & Alejandro Lillo \\
\hline & Guillermo Quintanilla & & & & Aldo González \\
\hline
\end{tabular}

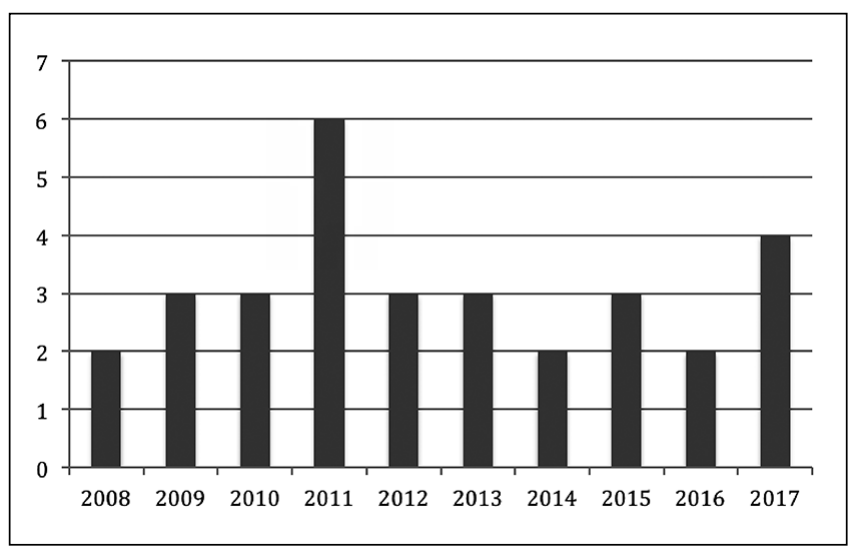

Gráfico 7. Becados formados anualemente en el Programa de Anestesiología de la Universidad Mayor 2008 - 2017.
La moderada holgura en las rotaciones que poseía en el Hospital Dipreca, se ha visto alterada por la incorporación a ese hospital de otro programa de inicio posterior, el de la Universidad Diego Portales, con el cual cohabita en dicho centro asistencial.

\section{UNIVERSIDAD DEL DESARROLLO}

Jefe de Programa: Dr. Juan Abarca

Acreditación: El programa se encuentra acreditado por APICE, según consta en el Acuerdo $\mathrm{N}^{\circ} 56$ de dicha entidad, otorgándole un período de vigencia de 3 años, hasta el 16 de noviembre de 2015.

Becados en tercer año: 2

Becados en segundo año: 3

Becados en primer año: 2

La Tabla 10 es la nómina de todos los egresados del Programa de Anestesiología de la Universidad del Desarrollo, desde el año 2012 hasta el año 2014.

El Gráfico 8, muestra la progresión en el número de becados en formación anual a partir del año 2012.
El programa tiene su campo clínico base en la Clínica Alemana de Santiago y utiliza como campo clínico asociado el Hospital Padre Hurtado. Además, complementa su malla curricular con el uso de los campos clínicos del Instituto Nacional del Tórax en anestesia para cirugía torácica, Instituto de Neurocirugía en anestesia neuroquirúrgica y Hospital Luis Calvo Mackenna en anestesia pediátrica.

Son probablemente los cupos en estos campos clínicos complementarios - que por cierto son limitados - los que han hecho que las autoridades de este programa tengan una actitud muy prudente en cuanto al aumento de las vacantes anuales.

\section{UNIVERSIDAD DE LOS ANDES}

Jefe de Programa: Dr. Mario Portilla Acreditación: El programa se encuentra acreditado por APICE, según consta en el Acuerdo $\mathrm{N}^{\circ} 27$ de dicha entidad, otorgándole un período de vigencia de 3 años, hasta el 12 de agosto de 2014, por lo cual en estos momentos se encuentran en trámite de re acreditación, habiéndose ya realizado la visita de la Comisión de Pares Evaluadores. 
Tabla 10. Nómina de egresados del programa de anestesiología de la Universidad del Desarrollo 2008 - 2014

\begin{tabular}{|ll|}
\hline 2012 & $\begin{array}{l}\text { Bernardita Valenzuela } \\
\text { J. Sebastián Silva }\end{array}$ \\
\hline
\end{tabular}

\begin{tabular}{|ll|}
\hline 2013 & Cristian Muñiz \\
& Pamela Valentin \\
\hline
\end{tabular}

\section{Diego Buckuk \\ Daniela Subiabre}

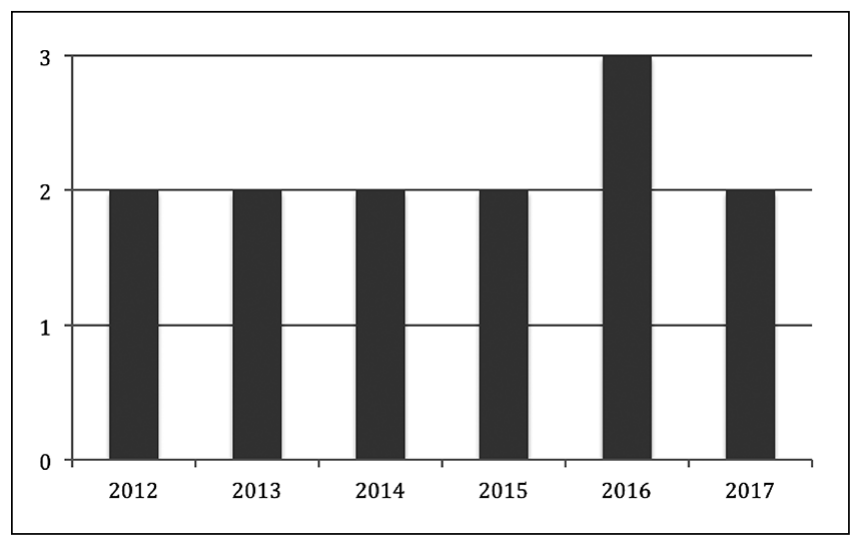

Gráfico 8. Becados formados anualmente en el Programa de Anestesiología de la Universidad del Desarrollo 2012 - 2017.

Tabla 11. Nómina de egresados del programa de anestesiología de la Universidad de los Andes 2008 - 2014

\begin{tabular}{|ll|}
\hline 2012 & Daniela Aybar \\
& Magdalena Cruz \\
& Rosita Marín \\
\hline
\end{tabular}

\begin{tabular}{|c|c|}
\hline \multirow[t]{3}{*}{2013} & Bernardita Quijano \\
\hline & J. Pablo Rodríguez \\
\hline & Esteban Arriagada \\
\hline
\end{tabular}

\begin{tabular}{|ll|}
\hline 2014 & Karen Müller \\
& Macarena Ávila \\
& Paula Penroz \\
\hline
\end{tabular}

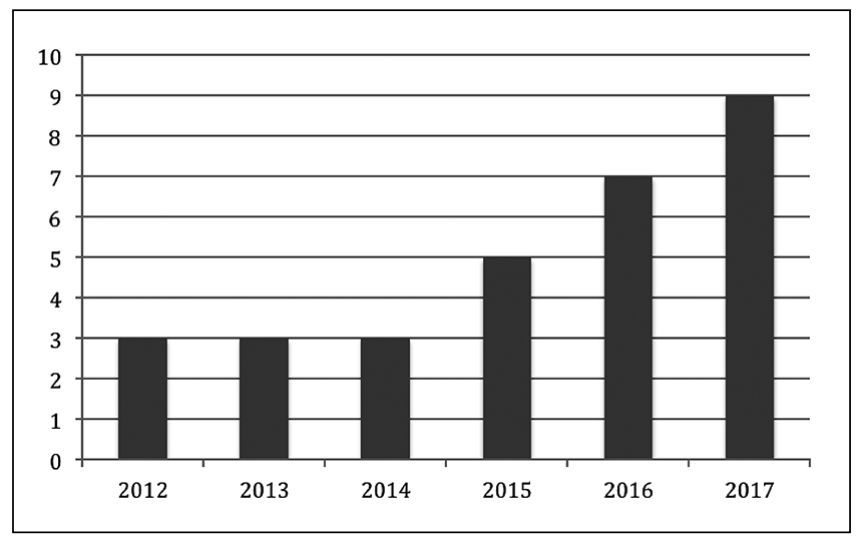

Gráfico 9. Becados formados anualmente en el Programa de Anestesiología de la Universidad de los Andes 2012 - 2017.

Becados en tercer año: 5

Becados en segundo año: 7

Becados en primer año: 9

La Tabla 11 es la nómina de todos los egresados del Programa de Anestesiología de la Universidad de los Andes, desde el año 2012 hasta el año 2014.

El Gráfico 9, muestra la progresión en el número de becados en formación anual a partir del año 2012 .
El programa tiene dos campos clínicos base en las Clínicas Santa María y Dávila, donde los becados desarrollan prácticamente toda su malla curricular. Estos campos clínicos ofrecen acceso a una casuística suficiente como para explicar el aumento progresivo del número de becados aceptados por año, manteniéndose independiente de la necesidad de acceso a otros campos clínicos complementarios. 


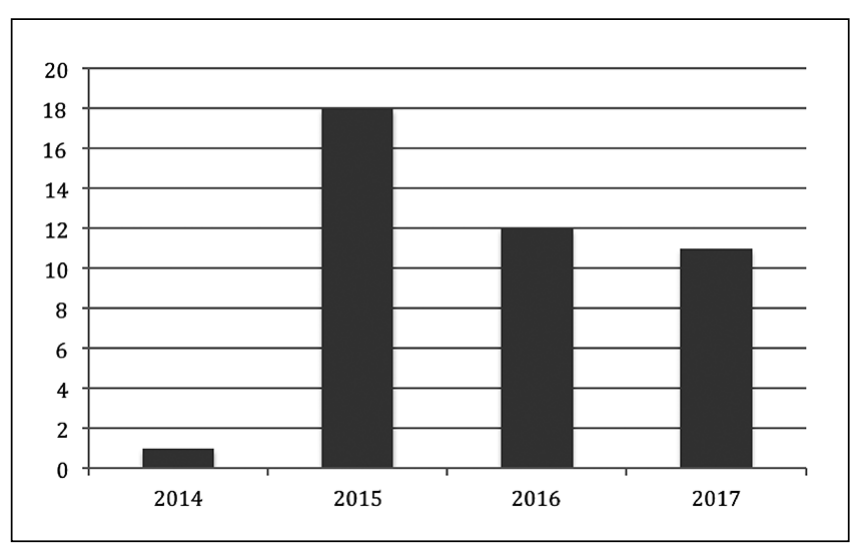

Gráfico 10. Becados formados anualmente en el Programa de Anestesiología de la Universidad Diego Portales 2014 - 2017.

\section{UNIVERSIDAD DIEGO PORTALES}

Jefe de Programa: Dr. Ricardo Lathrop

Acreditación: El programa no se ha presentado a acreditación ante APICE

Becados en tercer año: 18

Becados en segundo año: 12

Becados en primer año: 11

El programa tiene un solo egresado en el año 2014, que es el Dr. Sebastián Acosta.

El Gráfico 10, muestra la progresión en el número de becados en formación anual a partir del año 2014.

Este programa inició su funcionamiento en el Hospital Dipreca, en el año 2011, con una oferta de 3 cupos anuales, pese a que desde el año 2005 dicho campo clínico ya se encontraba ocupado por el programa de la Universidad Mayor. De esa primera generación solamente egresó el Dr. Acosta, ya mencionado.

A partir de su segundo año de funcionamiento, el programa decide realizar un brusco aumento en su capacidad formadora, incorporando como campos clínicos a los hospitales del Salvador, Barros Luco y San Borja Arriarán, en cada uno de los cuales ofrece 5 cupos, mientras mantiene los 3 ya existentes en el Hospital Dipreca.

De este modo, su segunda generación (aún en formación) se encuentra conformada por 18 becados, distribuidos en estos cuatro hospitales.

La suma de los residentes que hoy cursan la beca en sus diferentes promociones, convierte a este programa con 41 becados al cabo de 4 años de funcionamiento, en el segundo más grande del país, solamente por debajo del de la Universidad Católica.

Teniendo en cuenta este gran número de becados, mientras se ejecuta, el programa ha tenido que conseguir rotaciones por distintos campos clínicos, para poder completar la malla curricular. Es así como han logrado resolver algunas de ellas de la siguiente forma:

Anestesia pediátrica: Los becados pueden realizar su rotación en el Hospital San Borja Arriarán, en el Hospital Exequiel González Cortés o en la Clínica Dávila, decidiendo la jefatura del programa a cuál de estos campos es enviado cada uno.

Anestesia cardiovascular: Los becados pueden realizar su rotación en la Fundación Favalloro en Buenos Aires (que recibe 3 ó 4 becados en paralelo) o en el Hospital San Borja Arriarán si tienen problemas de cupo, familiares o presupuestarios para viajar fuera de Chile.

Anestesia en cirugía de tórax: Los becados también cuentan con la alternativa de la Fundación Favalloro o en su defecto el Hospital Barros Luco, teniendo en cuenta las mismas variables.

Anestesia Neuroquirúrgica: El programa cuenta con cupos en el Instituto de Neurocirugía o en el Hospital Barros Luco.

Para la redacción de este artículo no se inquirió a los distintos programas respecto a la malla teórica que se les ofrece a los becados durante su formación, sin embargo, llama la atención que existen becados de este programa que se encuentran cursando por cuenta propia, el Diplomado en Fundamentos de la Anestesiología Aplicada dictado por la Pontificia Universidad Católica de Chile.

\section{UNIVERSIDAD DE LA FRONTERA}

Jefe de Programa: Dr. Hugo Becerra

Acreditación: El programa se encuentra acreditado por APICE, según consta en el Acuerdo № 109 de dicha entidad, otorgándole un período de vigencia de 3 años, hasta el 4 de julio de 2017. 


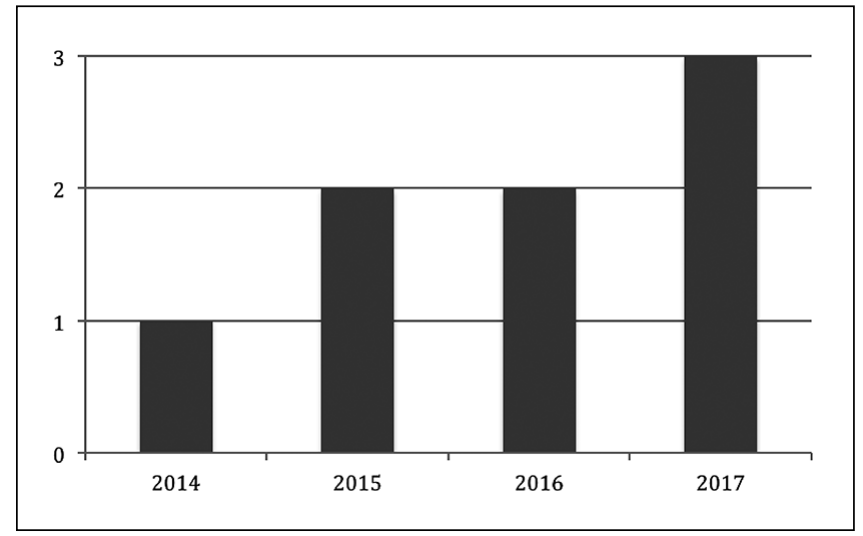

Gráfico 11. Becados formados anualmente en el Programa de Anestesiología de la Universidad de La Frontera 2014 -2017.
Becados en tercer año: 2

Becados en segundo año: 2

Becados en primer año: 3

El programa tiene un solo egresado en el año 2014, que es el Dr. Raúl Silva.

El Gráfico 11, muestra la progresión en el número de becados en formación anual a partir del año 2014.

El programa se desarrolla fundamentalmente en el Hospital Regional de Temuco, con complemento de la Clínica Alemana de Temuco, lográndose con ello el desarrollo de todas las rotaciones en condiciones suficientes en relación a la cantidad de becados en formación.

\section{UNIVERSIDAD AUSTRAL DE CHILE}

Jefe de Programa: Dr. Guillermo Lema - Dr. Bernardo Jorquera

Acreditación: El programa se encuentra acreditado por APICE, según consta en el Acuerdo $\mathrm{N}^{\circ} 94$ de dicha entidad, otorgándole un período de vigencia de 3 años, hasta el 31 de enero de 2017.

Becados en tercero año: No tiene Becados en segundo año: 2

Becados en primer año: 3

El programa aún no tiene egresados.

El desarrollo de este proyecto es bastante interesante, pues presenta características dignas de tener en cuenta:

- El proyecto se desarrolla al amparo de una Universidad con dilatada experiencia en el ámbito de la medicina.

- Aún así, la Universidad decide involucrar como jefe del programa a un docente externo con amplia experiencia académica - el Dr. Guillermo Lema - a quien otorga horas docentes y a quien categoriza como Profesor Titular. De este modo, coloca a la cabeza del programa a un docente cuya categorización académica no es un simple ejercicio administrativo, si no un proceso que toma en cuenta su experiencia docente.

- Considerando que el Dr. Lema no puede tener presencia continua en la sede del programa, nombra a un coordinador local - el Dr. Bernardo Jorquera - también anestesista de vasta trayectoria, pero con menor experiencia académica, como director local del proyecto.

- El programa inicia su funcionamiento ya preparado y dispuesto a postular a su acreditación durante el primer año de actividad y realiza dicho proceso en un nivel tal, que logra su acreditación ante APICE apenas transcurridos algunos meses desde que la primera promoción de becados ingresó.

- El programa conserva firme su intención de mantener el número de becados por año, para solo aumentar moderadamente ese número una vez egresada su primera promoción, siempre teniendo en cuenta la disponibilidad asegurada de rotaciones como para obtener un adecuado resultado docente.

Según el Dr. Lema, las pasadas que constituyen cuello de botella son anestesia en cirugía torácica y cardiovascular, para lo cual el programa se inició con convenios de postgrado ya establecidos con el Hospital Regional de Temuco, donde se realizarán dichas rotaciones.

Otro aspecto importante es que los primeros 4 becados en formación serán contratados por el propio Hospital de Valdivia, como una forma de aumentar la plana docente. 


\section{UNIVERSIDAD SAN SEBASTIÁN}

Jefe de Programa: Dr. Marco Balkenhol

Acreditación: El programa no se ha presentado al proceso de acreditación.

Becados en tercer año: No tiene

Becados en segundo año: 3

Becados en primer año: 2

El programa aún no tiene egresados.

Este programa se desarrolla íntegramente en el Hospital Regional de Puerto Montt, donde trabajan 9 anestesiólogos.

Según señala el Dr. Balkenhol, tratándose de un hospital de referencia del sur del país, su casuística permite cubrir sin problemas las rotaciones de anestesia pediátrica con un número adecuado de anestesia en neonatos, anestesia en cirugía de tórax $\mathrm{y}$ anestesia neuroquirúrgica.

En consecuencia, el problema a solucionar es la disponibilidad de campo clínico para desarrollar la rotación de anestesia cardiovascular, la cual pese a que el programa ya está funcionando aún no está definida, barajándose las posibilidades del Hospital Grant Benavente en Concepción, el Hospital Sótero del Río en Santiago o la Fundación Favalloro en Buenos Aires.

El Dr. Balkenhol señala también que las primeras promociones de becados deben ser contratadas en el propio Hospital de Puerto Montt - recientemente inaugurado - cuya dotación de 15 pabellones quirúrgicos hace necesario ampliar la planta de anestesistas.

\section{UNIVERSIDAD CATÓLICA DEL MAULE}

Jefe de Programa: Dr. Sergio Latrach

Acreditación: El programa no se ha presentado al proceso de acreditación

Becados en tercer año: No tiene

Becados en segundo año: 2

Becados en primer año:

El programa aún no tiene egresados.

Este programa, que se desarrolla en el Hospital de Talca, está inmerso dentro del Plan Maule de formación de 100 especialistas para esa región y cuenta con el firme apoyo económico del Servicio de Salud Maule, el que ha destinado cuantiosos recursos a la formación de los especialistas necesarios para la Región.

Ejemplo de ello es este programa, que para solucionar su dificultad en el acceso a las rotaciones de anestesia cardiovascular, pediátrica y neuroquirúrgica, ha establecido un convenio con la Uni- versidad de Lovaina en Bélgica, por lo cual estos becados desarrollan un año completo de su beca en Bélgica, donde realizan rotaciones de cuatro meses por anestesia cardiovascular, cuatro meses por anestesia pediátrica y cuatro meses por anestesia neuroquirúrgica.

Del mismo modo, el Servicio de Salud Maule financió la inscripción de estos becados en el Diplomado Fundamentos de la Anestesiología Aplicada organizado por la Universidad Católica, como recurso para ofrecerles un programa teórico adecuado.

\section{UNIVERSIDAD CATÓLICA DE LA SANTÍSIMA CONCEPCIÓN}

\section{Jefe de Programa: Dr. Juan Carlos Gómez}

Acreditación: El programa no se ha presentado al proceso de acreditación.

Becados en primer año: 3

El programa aún no tiene egresados

Este programa, que inició sus funciones en abril de 2014, se desarrolla principalmente en el Hospital Herminda Martin de Chillán, donde participan en docencia los 12 miembros del Servicio de Anestesiología, de los cuales la Universidad contrató a 8 para efectos de su ejecución, a quienes inicialmente capacitó a través de diplomados en docencia médica.

Según señala el Dr. Gómez poseen campo clínico para el desarrollo de prácticamente toda la malla curricular en el propio Hospital Herminda Mártin, excepto anestesia pediátrica compleja del neonato $\mathrm{y}$ anestesia cardiovascular, rotaciones que tienen resueltas a través de un convenio con el Hospital Clínico de la Pontificia Universidad Católica de Chile, que se encargaron de gestionar previo al inicio de la beca.

En este punto, el Dr. Gómez es enfático en señalar que ellos demoraron el inicio del programa por al menos un año y medio, hasta no contar con toda la malla curricular resuelta a través del acceso a los campos clínicos correspondientes. Del mismo modo, señala que otra de las exigencias a la Universidad previo a la recepción de los primeros residentes, fue cumplir con al menos el $90 \%$ de los requisitos impuestos por APICE para la acreditación del programa, puesto que pretenden presentarlo en el corto plazo a este trámite, de manera que los primeros residentes egresen con su certificación definitiva.

Se debe destacar además que por exigencias de las malla curricular, los becados de este programa también deben rendir y aprobar en su primer año de 
beca el Diplomado Fundamentos de la Anestesiología Aplicada de la Universidad Católica.

\section{UNIVERSIDAD ANDRÉS BELLO}

Jefe de Programa: Dra. Carmen Yáñez

Acreditación: El programa no se ha presentado al proceso de acreditación.

Becados en primer año: 3

El programa aún no tiene egresados.

Este programa se desarrolla en el Hospital Las Higueras de Talcahuano, pero según la Dra. Carmen Yáñez deben encontrar campo clínico donde efectuar algunas rotaciones en que el Hospital no posee casuística:

Anestesia pediátrica: El Hospital las Higueras la desarrolla en número suficiente para la realización de una rotación adecuada.

Anestesia cardiovascular: El Hospital las Higueras también tiene una casuística suficiente como para el desarrollo de la rotación.

Anestesia neuroquirúrgica: La deben conseguir en otro campo clínico. Según señala la Dra. Yáñez "la universidad se está encargando de conseguir la rotación" y agrega que existe un convenio entre la Universidad Andres Bello y el Instituto de Neurocirugía. Pese a esto, ella dice saber que el Servicio de Anestesia del Instituto de Neurocirugía tiene su capacidad docente plenamente colmada por el paso de varias otras universidades, por lo cual está presionando para obtener una respuesta definitiva.

Anestesia en cirugía de tórax: También deben conseguir esta rotación. Obviamente han pensado en el Instituto Nacional del Tórax, pero la Dra. Yáñez también dice estar consciente de que dicho Instituto se encuentra en estos momentos con su capacidad formadora colmada, incluso recibiendo dos becados por pabellón provenientes de otras universidades.

En definitiva, el programa inició su funcionamiento y recibió a su primera promoción de becados sin saber donde podrán enviar a estos becados cuando les corresponda realizar algunas rotaciones específicas.

\section{SERVICIO DE SALUD METROPOLITANO SUR-ORIENTE}

Jefe de Programa: Dr. Edwin Coronel Acreditación: El programa no se ha presentado al proceso de acreditación.

Becados en primer año: 2
El programa aún no tiene egresados.

Este programa, que inició su funcionamiento este año 2014 y que se encuentra radicado en el Hospital Sótero del Río, es lo que se suele denominar una "beca CONACEM", vale decir un programa no sustentado en ninguna universidad y en consecuencia "no acreditable" de acuerdo a las exigencias de APICE, por lo que sus egresados deberán rendir el examen de CONACEM para poder certificarse como especialistas.

De acuerdo a lo que señala el Dr. Coronel, los anestesiólogos del Hospital Sótero del Río han comprometido su esfuerzo en el desarrollo de este programa, incluyendo aquellos que se desempeñan en la Empresa Morpheus, que abastece de anestesistas a un porcentaje importante de los pabellones de la Institución. Se debe señalar sin embargo, que el trabajo de los especialistas de Morpheus en el Hospital, depende de una licitación que se realiza cada cierto tiempo y que eventualmente podría significar el cambio de prestador, con el consiguiente cambio del contingente de docentes.

Respecto a las rotaciones ya conocidas como cuello de botella, el Dr. Coronel señala que estas se encuentran parcialmente resueltas de la siguiente forma:

Anestesia pediátrica: Se programa realizar la rotación en el hospital pediátrico perteneciente al mismo complejo hospitalario (ex Hospital Josefina Martínez).

Anestesia en cirugía cardiovascular: Se realizará en el Hospital Sótero del Río.

Anestesia neuroquirúrgica: Se realizará en el Hospital Sótero del Río.

Anestesia en cirugía de tórax: No existe solución a esta demanda por el momento.

En consecuencia, este es otro programa que inició sus funciones y recibió a su primera promoción de becados sin tener certeza donde se desarrollarán algunas rotaciones, en etapas más avanzadas de la beca.

\section{CONCLUSIONES}

Este artículo ha revisado la historia de la formación de anestesistas a partir de la conformación de los primeros programas estructurados, en relación a las universidades más tradicionales del país.

A partir de ahí, se ha analizado la progresión que ha tenido dicho proceso en el transcurso de los años, tanto a nivel global como en cada uno de esos programas y los que se han ido agregando en el transcurso del tiempo.

Este análisis coloca en perspectiva cuál es la realidad de la formación de anestesistas en Chile y 
cómo este proceso puede llevar a resolver el déficit de especialistas que exhibe el país. Nosotros, los anestesistas, estamos obligados a conocer la envergadura del fenómeno, pero más obligada aún está la Sociedad de Anestesiología de Chile, que es la entidad que se debe interrelacionar con las autoridades políticas y universitarias, para como lo señalan sus estatutos en el punto 2: "Propender al progreso de la anestesia".

En mi opinión, este fenómeno de ampliación de la oferta de programas de anestesiología se ha iniciado sin vuelta atrás y probablemente es lo que corresponde para suplir la enorme demanda de especialistas que exhibe el país.

Sin embargo, no se requiere ser tan perspicaz para darse cuenta de la visión disímil que tienen las distintas universidades respecto a la forma de planificar y ejecutar los proyectos docentes. Esa dispersión no es más que un reflejo de la estructura de la propia educación superior en Chile y en consecuencia no debemos esperar que sean las universidades las que en forma equivalente pongan cordura y rigor al momento de abordar una tarea tan delicada como la formación de anestesistas para el país. Quienes nos debemos convertir en el garante de calidad de los programas, somos los anestesiólogos que participamos de cada proyecto.

En este artículo hemos comprobado como mientras algunos proyectos tienen la prudencia de iniciar sus actividades solo una vez que han asegurado el poder ofrecerle a sus residentes la malla curricular completa, otros parecen iniciar sus actividades siguiendo el principio popular que señala "en el camino se arregla la carga"; hemos comprobado como mientras algunos programas son celosos de que el aumento del número de becados en formación se ajuste en forma estricta a la disponibilidad de rotaciones y recursos docentes, otros nuevamente "en el camino arreglan la carga". El problema está cuando "en el camino no se puede arreglar la carga", porque en ese momento se les está mintiendo a los hospitales que recibirán a esos especialistas, a los propios becados y mucho más importante aún, a los pacientes.

El párrafo anterior no pretende ser una mirada pesimista del futuro de nuestra especialidad. Al concluir este artículo debo señalar que mi visión del fenómeno es más bien positiva, puesto que creo haber identificado en muchos jefes de programa, docentes realmente comprometidos con la calidad de los profesionales en formación. Las cifras están ahí para el análisis de todos y cada uno de ustedes. Ahora saben cuánto se forma y tienen algunos antecedentes para deducir cómo se forma.

Al finalizar, una palabra respecto a la responsabilidad que adquieren los propios becados al enfrentar este proceso. La "beca CONACEM" que se acaba de iniciar en el Hospital Sótero del Río, es entre otras cosas la respuesta de las autoridades del Servicio de Salud Metropolitano Sur-Oriente a la actitud de dos post becados de anestesiología de un prestigioso programa de formación, quienes luego de haber realizado su formación financiados por dicho Servicio de Salud, decidieron pagar la fianza y no devolver el tiempo asistencial comprometido, condición que ellos conocían desde el momento mismo en que postularon a la beca. Este fenómeno no es aislado y obliga a quienes nos desempeñamos como docentes de estos programas de especialidad no sólo a poner esfuerzos en instruir, entrenar y capacitar a los becados en el arte de la anestesiología, si no también a educarlos en forma integral, obteniendo de ellos actitudes de respeto y lo primero que ellos deben aprender a respetar es la palabra empeñada.

Correspondencia a:

Renato Chacón A.

rchacon@mi.cl 